\title{
A deflexão gravitacional da luz: De Newton a Einstein
}

The gravitational deflection of light: From Newton to Einstein

\author{
César H. Lenzi ${ }^{* 10}$, Pedro J. Pompeia ${ }^{1}$, Nelson Studart ${ }^{2,3}$ \\ ${ }^{1}$ Instituto Tecnológico de Aeronáutica, São José dos Campos, SP, Brasil \\ ${ }^{2}$ Universidade Federal do ABC, Santo André, SP, Brasil \\ ${ }^{3}$ Universidade Federal de São Carlos, São Carlos, SP, Brasil
}

Recebido em 04 de Setembro de 2019. Aceito em 09 de Setembro de 2019

\begin{abstract}
A comprovação do encurvamento da luz devido a efeitos gravitacionais constitui um importante marco na história da ciência, porque as medidas da deflexão da luz nas observações astronômicas durante o eclipse de 1919 confirmaram o valor previsto pela teoria da relatividade geral de Einstein, concluída em 1915. O fato teve uma repercussão extraordinária na imprensa, o que levou Einstein ao reconhecimento mundial e a se tornar o cientista mais midiático do século XX. No entanto, a deflexão da luz foi motivo de atenção desde Newton. Este artigo discute os avanços e cálculos realizados por predecessores de Einstein e detalha sua trajetória no desenvolvimento da teoria da relatividade até a determinação exata da deflexão gravitacional da luz.
\end{abstract}

Palavras-chave: deflexão da luz, história da física, relatividade geral.

Evidence of light bending due to gravitational effects is an important milestone in the history of science because measurements of light deflection in astronomical observations during the 1919 eclipse confirmed the value predicted by Einstein's theory of general relativity in 1915. This had an extraordinary repercussion in the press, which led Einstein to worldwide recognition and to becoming the most famous scientist in the non-scientific media of the twentieth century. However, light deflection had been a matter of attention since Newton. This paper discusses the advances and calculations performed by Einstein's predecessors and details his trajectory in the development of relativity theory until the exact determination of the gravitational deflection of light.

Keywords: light deflection, history of physics, general relativity.

\section{Introdução}

Em seus escritos, que incluem, além do Principia e do Óptica, muitas contribuições no Philosophical Transactions da Royal Society e trocas de correspondência com simpatizantes e antagonistas de suas ideias [1], Isaac Newton (1643-1727) sempre defendeu uma distinção clara entre as causas e as propriedades dos fenômenos. No caso da luz, as propriedades são as leis da reflexão e refração e a análise da luz branca. As hipóteses causais consistiam nos modelos da natureza da luz, ondas ou pequenas partículas, e da natureza das cores. Nas famosas "Questões" do Óptica [2], Newton tece considerações sobre a natureza corpuscular da luz, mas deixa claro que essas ideias não constituíam proposições provadas e sim hipóteses especulativas para pesquisa futura [3]. No entanto, seus seguidores transformaram muitas de suas ideias e teorias, em particular o tema da luz. Conforme ressaltam Silva e Moura [4], apenas seis anos após a publicação do Óptica, o verbete "Light" de Lexicon Technicum de John Harris (1666-1719) já afirmava que os raios de luz do modelo geométrico de Newton eram "pequenas partículas, realmente emitidas do corpo luminoso e refratadas por alguma atração, pela qual a luz e o corpo sobre o qual ela cai agem mutuamente um no outro, pois tais partículas ou corpúsculos serão transmitidos através de meios uniformes em linhas retas, sem qualquer inflexão, como os raios de luz fazem". (Harris, apud Silva e Moura [4].)

Seus seguidores imediatos, o reverendo John Michell (1724-1793) e Henry Cavendish (1731-1810), adotaram o modelo corpuscular para calcular a deflexão gravitacional da luz na tentativa de responder à Questão 1 do Óptica de Newton, "Os corpos não agem sobre a luz a distância e, por sua ação, não curvam os seus raios? E essa ação não é mais forte na distância menor?", que será discutida na seção 2 do presente artigo. Enfatiza-se, na seção 3, o papel relevante de Michell na deflexão gravitacional da luz, haja vista que ele se tornou mais conhecido na literatura de divulgação científica pela previsão, junto com Laplace, da existência dos buracos negros.

No entanto, foi um astrônomo alemão, Johann Georg von Soldner (1876-1833), quem primeiro publicou, em 1801, o cálculo do valor do desvio angular devido à força gravitacional exercida sobre um raio de luz ao passar por um corpo de grande massa, como o Sol. Um tratamento detalhado, e com pretensões didáticas, do trabalho de Soldner será dado na seção 4 .

*Endereço de correspondência: chlenzi@ita.br 
Independentemente, Cavendish também obteve esse valor, embora não tenha publicado o resultado, encontrado em rascunhos de sua autoria quando da compilação final de sua obra. Na seção 5, o valor obtido por Cavendish será calculado usando a bem estabelecida mecânica newtoniana e comparado com o resultado de Soldner. Será mostrado que os resultados diferem em virtude de os autores terem assumido diferentes condições de contorno para a velocidade da luz proveniente de uma fonte distante ao se aproximar do Sol. No entanto, os valores numéricos não diferem em primeira ordem. Assim, o resultado aproximado de 0,84 segundos de arco para a deflexão da luz ficou conhecido como o valor newtoniano.

A seção 6, a mais importante do artigo, trata da trajetória de Albert Einstein (1879-1955) desde o artigo seminal de revisão de 1907 para estabelecer, no contexto da relatividade geral, o valor correto da deflexão gravitacional da luz. Em artigo de 1911, Einstein descarta a visão corpuscular de Newton. O resultado, atualmente conhecido como newtoniano (1911) de Einstein foi obtido a partir de: a) a natureza ondulatória da luz ao usar o conceito de frente de ondas de Huygens e admitir que a velocidade da luz muda sua direção ao passar por um corpo massivo; b) o princípio de equivalência inferido da conclusão de Galileu de que a aceleração de um corpo na presença de um campo gravitacional independe de massa, estrutura e material do corpo; c) a lei da gravitação universal de Newton. Mas ainda Einstein não sabia que a gravidade não deve ser tratada como uma ação a distância entre corpos, mas sim como um efeito da deformação do espaço-tempo devido à presença de uma quantidade de matéria Somente com a versão final da relatividade geral em 1915, Einstein apresentou o valor de 1,75 segundos de arco, comprovado em medidas fotográficas nas observações dos astrônomos britânicos feitas durante o eclipse de 1919.

A seção 7 traz uma abordagem moderna ao problema de encurvamento da luz, tomando como base a primeira solução exata das equações de Einstein, apresentada em 1915 por Karl Schwarzschild (1873-1916). Apesar de não ser a abordagem tomada por Einstein em seu artigo de 1915, essa solução tem seu valor histórico, visto que confere à relatividade geral a confirmação de testes - tais quais o avanço do periélio de Mercúrio, tema que não é tratado neste trabalho - , além de trazer luz à previsão da existência de buracos negros e a outras questões importantes no desenvolvimento da cosmologia moderna tal qual a conhecemos.

Por razões de completeza e curiosidade sobre o tema, é interessante mencionar o papel de um dos líderes da revolução francesa, Jean Marat (1743-1793), que imaginou o encurvamento da luz das estrelas devido à gravitação 11 No livro Découvertes sur la lumiére [5], Marat afirma que

\footnotetext{
1 Marat, além de político revolucionário, foi médico e escritor, tendo passado 11 anos na Inglaterra. Fez experimentos para estudo de fenômenos ópticos e elétricos. Foi um dos primeiros tradutores para o francês do Óptica de Newton e mantinha boas relações com Benjamin Franklin. A Academia Francesa rejeitou fortemente suas ideias e os resultados de sua pesquisa [9].
}

"todos os corpos desconstroem a luz por atração [...]; a esfera de atração da luz depende da [...] densidade superficial, um fator de afinidade [...] e depende do quadrado do inverso da distância". No entanto, esse texto não deixa claro se essa atração é gravitacional ou simples atração por uma outra força [6]. Mais surpreendente é o texto escrito em Notions élémentaires d'optique [7] de 1784, mesmo ano da publicação de John Michell (1784):
Não há dúvida que os raios de luz mudam sempre de direção no mesmo meio, quando passam a determinada distância de um corpo. Encontram-se eles na esfera da atração? Eles se curvam até um certo ponto seguindo uma circunferência e se prolongam em seguida em linha reta.

Parece uma antecipação incrível, mas, segundo Osborne (1920), a conclusão é baseada em premissas falsas, como se observa na leitura do manuscrito.

\section{Isaac Newton: a Questão 1 do Óptica}

Na reunião conjunta da Royal Society e da Royal Astronomical Society, em 6 de novembro de 1919, após relato feito pelo astrônomo-real Frank Dyson, com a participação de Arthur Eddington (1882-1944) e Andrew Crommelin (1865-1939), responsáveis pelas expedições astronômicas a Príncipe e Sobral durante o eclipse solar de maio daquele ano, o presidente da Royal Society, Joseph John Thomson (1856-1940), que dirigia os trabalhos, assim se expressou [10] :

\begin{abstract}
Encaminho agora a discussão esta importante comunicação. Se os resultados obtidos indicassem apenas que a luz é afetada pela gravidade, já seria da maior importância. Newton sugeriu, de fato, essa ideia na primeira questão de seu livro Óptica, e sua sugestão teria presumivelmente levado à metade do valor. Mas esse não foi um resultado isolado; foi parte de todo um continente de ideias científicas que afetam os conceitos mais fundamentais da física [...] Esse é o resultado mais importante na teoria da gravitação desde os dias de Newton, e é adequado que seja anunciado em um encontro desta Sociedade, tão intimamente relacionada com ele.
\end{abstract}

Thomson estava se referindo àquela que viria a ser a mais famosa citação retirada do livro Opticks (1704, Book IV, Part 1) de Newton que diz (no original): "Query 1. Do not Bodies act upon Light at a distance, and by their action bend its Rays, and is not this action (caeteris paribus) strongest at the least distance?". Essa questão implica uma resposta afirmativa, como sugere Bernard Cohen no prefácio da edição moderna [11]:

"As especulações da Óptica não eram exatamente hipóteses, quando menos porque foram 
formuladas como questões. Mas se usarmos a própria definição de Newton, de que 'o que não for deduzido dos fenômenos deve ser chamado de hipótese', então elas são de fato hipóteses. O formato de questões pode ter sido adotado para evitar críticas, mas não esconde a extensão da crença de Newton. Cada uma das questões é formulada como uma negativa! Ou seja, Newton não pergunta de forma realmente interrogativa (Q. 1): 'Os corpos agem à distância sobre a luz...?' — como se não soubesse a resposta. Em vez disso, ele escreve: 'Não agem os corpos à distância sobre a luz...?' - como se soubesse bem a resposta — 'Ora, é claro que sim!'

Como defensor do modelo corpuscular da luz, Newton, cujo retrato é reproduzido na Fig. 1, poderia ter naturalmente pensado e até mesmo calculado a curvatura da luz em um campo gravitacional, uma vez que lidou com problemas muito mais intrincados na dinâmica celestial do que a curvatura da luz. Mas, não deve ser atribuído ao próprio Newton o "half-value" apontado na fala de Thomson.

\section{John Michell: óptica-dinâmica das estrelas e os buracos negros primordiais}

Uma carta do Reverendo John Michell (1724-1793), enviada a Henry Cavendish (1731-1810) e publicada em 1784, foi redescoberta nos anos 1970 e é reconhecida como tendo antecipado várias ideias astronômicas atuais [12]. A mais importante foi a tentativa de descobrir um método útil para determinar a massa de uma estrela. Michell considerava a óptica como a mecânica da força

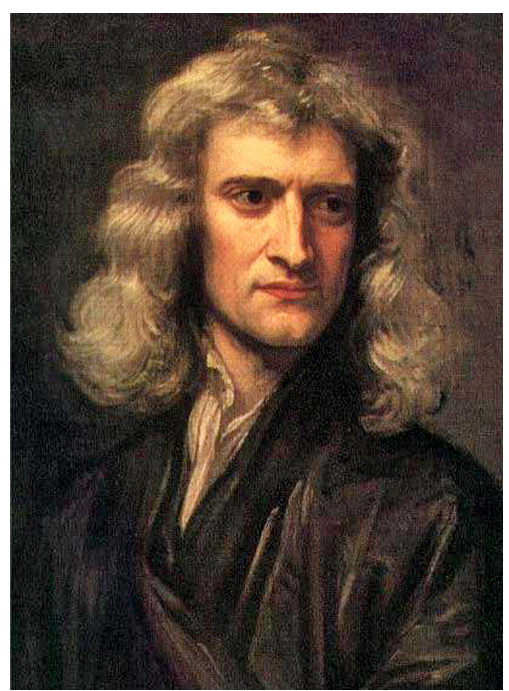

Figura 1: Retrato de Newton em 1689 (com 46 anos de idade). Fonte: Wikipedia. das partículas de luz. Sua hipótese física fundamental consistia em que [13]

partículas de luz são atraídas da mesma maneira que todos os outros corpos com os quais estamos acostumados; ou seja, por forças agindo na mesma proporção de sua vis inertiae, sobre as quais não pode haver dúvida razoável, a gravidade sendo, até onde sabemos, ou temos razão para acreditar, uma lei universal da natureza.

Michell foi um filósofo natural, seguidor incondicional de Newton, e sua linha de pesquisa, uma vez que a força da gravidade era conhecida, consistia na busca por novos efeitos estelares, terrestres e ópticos da gravitação: [13]

Entretanto, há uma força conhecida, a gravidade, que presumivelmente age sobre as partículas de luz e que fixa exatamente seus caminhos a distâncias razoáveis de outros corpos. O movimento gravitacional das partículas de luz não é, é claro, observado rotineiramente devido à sua grande velocidade. Ainda assim, um corpo extraordinariamente massivo como uma estrela poderia atrair sua própria luz com força bastante para causar uma diminuição na velocidade.

Ele presume, no artigo de 1784, que a força da gravidade atua sobre as partículas de luz e determina exatamente suas trajetórias a distâncias sensíveis dos corpos. O efeito gravitacional sobre o movimento das partículas de luz, é claro, não é normalmente observado devido à enorme velocidade da luz. Contudo, um corpo extremamente massivo, como o de uma estrela, poderia atrair sua própria luz com intensidade suficiente para causar uma diminuição expressiva em sua velocidade. Assim, a luz emitida por uma estrela teria sua velocidade reduzida devido à atração gravitacional, produzindo uma mudança observável na luz das estrelas.

Em seu trabalho de 1784, Michell considera uma estrela dupla com a maior no centro e a menor como seu satélite. Baseado nos Principia, ele afirma que características reais de uma estrela podem ser determinadas, caso sejam observadas a distância aparente, o diâmetro aparente da estrela central e o período de rotação do satélite, como havia antecipado no artigo anterior "Means of discovering the distance". Se, além disso, o valor da velocidade de diminuição da luz devido à gravidade da estrela é conhecido, então o tamanho real e demais propriedades da estrela ficam determinadas.

Nesse ponto, Michell calcula a velocidade de retardação da luz como fizera em artigo anterior publicado na History of optics de Joseph Priestley: ${ }^{2}$

\footnotetext{
$\overline{2}$ Michell havia feito estudos sobre a retardação gravitacional da luz das estrelas em trabalho anterior que apareceu em History of optics de Priestley, mas devido a "extreme difficulty, and perhaps impossibility" de detecção, não desenvolvera a ideia. Mas as poste-
} 
Michell fornece um exemplo de cálculo da diminuição da luz pela estrela central, desprezando os efeitos da rotação do satélite e aceleração da Terra sobre a luz. Como tradicionalmente na época, ele usa razões e proporções e o método discursivo. Aqui, vamos tratar do problema na notação moderna, de acordo com a reformulação desenvolvida por Eisenstaedt [14] e McCormmach [15].

Considere a emissão de uma partícula de luz de uma estrela de massa $M$, com a mesma densidade $\rho$ do Sol e raio $R=\alpha a$, em que $a$ é o raio do Sol e $\alpha$ é o número de vezes que o raio da estrela é maior do que o do Sol. A equação dinâmica estabelece que

$$
\frac{d^{2} r}{d t^{2}}=-\frac{G M}{r^{2}}
$$

em que $r$ é a distância da luz ao centro da estrela e $G$ é a constante gravitacional. A massa da partícula de luz é eliminada, admitindo que todos os corpos sofrem a mesma aceleração da gravidade. A massa que interessa a Michell é, portanto, a massa $M$ da estrela.

Uma simples integração resulta na expressão para a velocidade:

$$
c_{\infty}=c \sqrt{\left(1-\frac{8 \pi G \rho(\alpha a)^{2}}{3 c^{2}}\right)},
$$

em que $c_{\infty}$ é a velocidade da luz a uma distância infinita da estrela e $c$ a velocidade da luz na superfície da estrela.

A Eq. (2) fornece a velocidade de escape $c=v(a)$, que é a velocidade de queda livre, ou seja, a velocidade que a partícula de luz adquiriu caindo de uma distância infinita até parar, ou seja $c_{\infty}=0$. Se a estrela é o Sol, obtém-se

$$
\frac{v(a)}{c}=\sqrt{\frac{8 \pi G \rho a^{2}}{3 c^{2}}}=\frac{1}{497} .
$$

Substituindo na Eq. (3) para $c_{\infty}$, a razão entre a velocidade reduzida no infinito e a que não sofre diminuição é dada por

$$
\frac{c_{\infty}}{c}=\sqrt{1-\frac{\alpha^{2}}{(497)^{2}}} .
$$

Em seu exemplo, Michell tomou $c_{\infty} / c=19 / 20$, o que dá $a=155$, ou seja, o raio da estrela é 155 vezes o raio do Sol. Se a densidade da estrela é a mesma do Sol, sua massa vale 3.738.308 massas do Sol.

Se no exemplo acima o raio da estrela fosse 497 vezes o do Sol, em vez de 155 vezes, a velocidade da luz no infinito seria zero. Caso o raio fosse maior do que 497, a luz não iria até o infinito, pararia em algum lugar e

riores observações de John Herschel de uma grande quantidade de estrelas duplas o motivaram a seguir adiante na proposta, conforme explicitado na carta particular a Cavendish [12]. Isso demonstra que o trabalho pioneiro de Michell acerca da ação gravitacional sobre a luz é de 1772, portanto 11 anos antes do artigo encaminhado a Cavendish para publicação. retornaria até a estrela. Uma estrela desse tamanho foi subsequentemente chamada de "buraco negro newtoniano".

Em seguida, Michell calcula a separação da estrela dupla e a distância da Terra usando, sem citar, a lei dos períodos de Kepler tanto para a estrela central e seu satélite quanto para o Sol e seu satélite Terra. Alguns números obtidos: 64 foi o número escolhido para o período da estrela dupla, a separação da estrela dupla é 2.483 vezes a distância Terra-Sol (UA). Se a distância aparente entre as estrelas é conhecida por nós - diga-se que seja da ordem de 1 segundo -, a estrela estaria a 512.196.750 UA, que corresponde a milhares de vezes mais distante que o limite inferior dado pelas observações de Bradley para a distância das estrelas [15].

O método de Michell enfrentou dificuldades óbvias. Entre outras, a ausência de medição de diâmetros de estrelas ou períodos de estrelas duplas. Não havia sido tentada a experiência de medida da diminuição da velocidade da luz e ainda a hipótese de que a densidade da estrela seria a mesma do Sol é arbitrária.

A técnica proposta por Michell para medir a alteração na refrangibilidade consistia em apontar um prisma para uma estrela dupla de tal modo que a borda principal do prisma formasse um ângulo reto com a linha que liga as estrelas, esperando que a luz com menor velocidade vinda da estrela central fosse refratada mais que a luz da menor estrela giratória. Comparando as imagens refratadas de diferentes estrelas, ele poderia determinar a diferença em suas gravidades superficiais e a partir daí suas respectivas massas [12].

Ele também usou o conceito de "velocidade de escape" e achou que essa velocidade crítica poderia ser usada para determinar massa e tamanho da estrela e imaginou que, se uma estrela fosse tão massiva, e sua gravidade proporcional a sua massa, a velocidade de escape seria equivalente à velocidade da luz. Em suas palavras: [13]

Se uma esfera com a mesma densidade do Sol tivesse seu semidiâmetro na proporção de quinhentos para um, e supondo que a luz seja atraída por outros corpos pela mesma força na proporção de sua [massa], toda luz emitida por tal corpo seria forçada a voltar a ele, por sua própria gravidade.

Michell sugeriu, afinal, que estrelas suficientemente densas e massivas poderiam parar completamente a luz que emitiam e se tornariam invisíveis, as "estrelas escuras". Achou que seria possível detectar indiretamente essas estrelas escuras se elas tivessem "gêmeas" (estrelas duplas) circulando ao redor. Como mostrado acima, essa observação de Michell é hoje amplamente citada na divulgação da história dos buracos negros, nome originalmente proposto por John Wheeler. E, em um dos mais singulares projetos da história da ciência, a imagem de um buraco negro foi obtida em 2019 ${ }^{3}$

\footnotetext{
3 Poucos anos após esse extraordinário insight de Michell, PierreSimon Laplace propôs um conceito similar de luz sendo presa por
} 
A diminuição da luz por efeito gravitacional não foi descoberta nem poderia ser observada, uma vez que a luz não se comporta como objetos comuns que alteram suas velocidades de acordo com as forças que atuam sobre eles. Contudo, essa anomalia só foi entendida em outro contexto acerca da natureza da luz. Mas, na época de Michell e segundo a teoria newtoniana, o esquema era perfeitamente coerente e passível de experimentação.

\section{Johann von Soldner: primeiro resultado publicado}

Em 1801, aparece o artigo "Sobre o desvio de um raio luminoso de seu movimento em linha reta devido à atração de um corpo celestial que passa próximo" no periódico Astronomisches Jahrbuch [16]. O objetivo é "desenvolver, a partir da teoria, isto é, das propriedades gerais e interações da matéria, todas as circunstâncias que possam influenciar a posição verdadeira ou mediana de um corpo celeste, de modo que se possa derivar todo o benefício que uma boa observação é capaz de oferecer". Afirma, de início, que já haviam sido observados desvios consideráveis da luz, como na aberração estelar, mas que podem existir outros desvios que são tão pequenos que tornam difícil decidir se são verdadeiros ou erros de observação. Seria o caso do desvio do raio luminoso quando passa perto de um corpo celestial.

O autor do artigo, Johann Georg von Soldner (17761833), cujo o retrato encontra-se na Fig. 2, não era um cientista profissional, mas um autodidata sem educação formal, assistente-aprendiz de Johann Elert von Bode, o



Figura 2: Retrato de Johann von Soldner, primeiro diretor do Observatório de Bogenhausen, em 1816. (Domínio público.)

objetos com enorme gravidade no livro Exposition du système du monde, publicado em 1796. Aqui, Laplace afirma que a luz de uma estrela cujo diâmetro fosse 250 vezes o diâmetro do Sol e cuja densidade fosse igual à da Terra seria presa em seu campo gravitacional. Esse livro motivou Soldner a determinar o encurvamento da luz pelo Sol em 1801. astrônomo real da Prússia e editor do Astronomisches Jahrbuch. O artigo não teve nenhum impacto no mundo científico. Ficou esquecido até mesmo por seus biógrafos, que não foram nem astrônomos nem cosmólogos.

Stanley Jaki, tradutor do artigo para o inglês, releva dois aspectos importantes do artigo de Soldner [17]. Um refere-se a seu excepcional talento de, influenciado por Laplace, "submeter a ideia de corpos centrais ao teste da física matemática". O outro seria sua "insistência rigorosa no caráter mecânico de todos os processos", que o fez admitir que a luz estaria sujeita à atração gravitacional como qualquer outro fenômeno material. Ressalte-se que a questão da deflexão da luz teria sido profundamente relevante para a física e a cosmologia na época. Uma medida da deflexão, como calculada por Soldner, teria reforçado a teoria corpuscular de Newton em confronto com a teoria ondulatória revivida pelo trabalho de Thomas Young no mesmo ano, 1801, do artigo de Soldner. Se a luz fosse uma propagação ondulatória, não deveria haver deflexão, mas essa conclusão mostrou-se equivocada pelo trabalho de Einstein de 1911.

Uma reimpressão parcial do artigo de Soldner só ocorreu em 1921 em artigo de Lenard, que o usou parcialmente para denegrir Einstein em sua campanha antissemita [18]. Essa nenhuma repercussão explica a ausência de referência a ele nos clássicos relatos de relatividade de H. Weyl (1918), M. Born (1920), A. S. Eddington (1920), W. Pauli (1921) e L. Silberstein (1922). Nos relatos populares sobre a relatividade de Einstein, Soldner nunca é mencionado.

Nesta seção, o trabalho de Soldner é revisitado devido a sua relevância para a História da Ciência, com a exposição, que pretende ser didática, do trabalho em que foi feita a primeira previsão publicada da deflexão gravitacional da luz na teoria newtoniana.

Interessante assinalar que o alemão Soldner se afasta da tradição britânica dos Principia na abordagem do problema, trocando as razões e proporções usadas, por exemplo, na exposição de Michell e Cavendish, por equações diferenciais e a notação francesa do Traité de mécanique céleste de Laplace. ${ }^{4}$ [17]:

De início, Soldner apresenta suas considerações iniciais

Antes de abordar a investigação em si, farei mais alguns comentários que facilitarão o cálculo. Como inicialmente determinarei apenas a deflexão máxima, considerarei que o raio de luz passa horizontalmente à superfície do corpo atraente no ponto de observação, ou presumirei que a estrela da qual vem o raio de luz é observada ao nascer no horizonte.

Soldner admite que, em um determinado instante, um raio de luz emerge. Contudo, devido à atração gravitacional, a luz é desviada, de forma curvilínea, no ponto $M$, seguindo, subsequentemente, ao longo de $\overline{M Q}$ A distância entre a frente de onda e o centro de atração da força

\footnotetext{
$\overline{4}$ Por exemplo $\mathrm{d}^{2} \mathrm{x} / \mathrm{dt}^{2}$ é escrito como $\mathrm{ddx} / \mathrm{dt} 2$.
} 


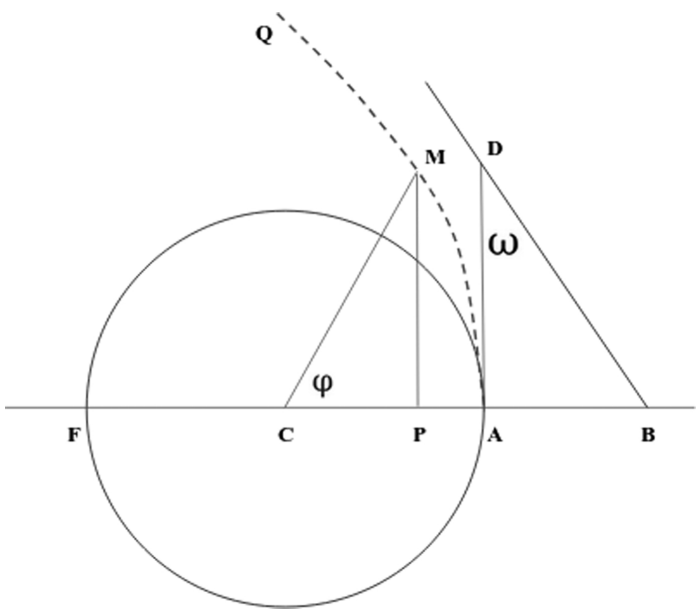

Figura 3: Versão da figura do artigo original de Soldner. A linha tracejada representa a trajetória de um raio de luz emitido do ponto $A$ na superfície da fonte. $\mathrm{O}$ ângulo $\varphi$ mostra a posição angular da frente de onda em um instante do tempo qualquer, e o ponto $C$ representa o centro da fonte gravitacional. O símbolo $\omega$ representa o desvio angular do raio de luz, devido à atração gravitacional. Para melhor compreensão dos símbolos, leia a nota de rodapé 5 .

$C$ é dada por $\mathbf{r}=(x, y)$ e a posição angular representada por $\varphi$.

Soldner considera que a intensidade da força de atração gravitacional que desvia o raio de luz é dada por $2 g r^{-2}$, em que $g=G M / 2$, sendo $M$ a massa da fonte e $G$ a constante de gravitação universal. ${ }^{5}$ Portanto, a decomposição sob os eixos $x=\overline{F B}$ e $y=\overline{F M}$ resulta em

$$
\frac{d^{2} x}{d t^{2}}=-2 g r^{-2} \cos \varphi
$$

e

$$
\frac{d^{2} y}{d t^{2}}=-2 g r^{-2} \operatorname{sen} \varphi
$$

Das Eqs. (5) e (6) é possível obter o seguinte sistema em coordenadas polares:

$$
\begin{gathered}
\frac{d}{d t}\left(r^{2} \frac{d \varphi}{d t}\right)=0 \\
\frac{d^{2} r}{d t^{2}}-r\left(\frac{d \varphi}{d t}\right)^{2}=-\frac{2 g}{r^{2}} .
\end{gathered}
$$

\footnotetext{
5 A consideração feita neste ponto é uma tradução didática do realizado por Soldner em seu artigo. De fato, no artigo original, a constante $g$ faz referência à própria constante de gravitação universal em um produto com a massa da fonte. Certamente Soldner sabia que o potencial gravitacional não é proporcional a $2 g$, como a leitura pode nos faz pensar. Sobre essa questão, Jaki [17] explica que Soldner obviamente sabia que o desvio da luz seria igual a $2 \times \omega$, ou seja, o símbolo da Fig. 3 mais sua imagem espelho, se considerada a fonte luminosa localizada no infinito. Tal interpretação, apesar de não explícita no artigo original, é consistente e justifica o uso do fator multiplicativo na obtenção dos resultados.
}

A Eq. (7) reflete a conservação do momento angular do sistema. Apesar de não discutir esse aspecto, Soldner nos recorda que o termo $r\left(r \frac{d \varphi}{d t}\right)$ equivale ao dobro da área do triângulo infinitesimal varrido pelo vetor $\vec{r}$ no intervalo infinitesimal de tempo $d t$. Deve ser notado ainda que, no instante em que o raio de luz é emitido do ponto A, $r=\overline{C A}$ e que o produto $v \overline{C A}$ em que $v$ é a velocidade da luz ao longo da trajetória, também equivale ao dobro da área do mesmo triângulo infinitesimal. Portanto é válido escrever

$$
\frac{d \varphi}{d t}=\frac{v \cdot R}{r^{2}}
$$

em que $R=\overline{C A}$. Aqui, é importante mencionar que Soldner assume $\overline{C A}=1$, oque pode levar a interpretações dimensionais equivocadas. No decorrer desta revisão, a opção foi considerar $R$ nas equações, que, para fins práticos, representa o raio da fonte.

Substituindo a Eq. (9) na Eq. (8) e integrando, obtémse

$$
\left(\frac{d r}{d t}\right)^{2}+\frac{R^{2} v^{2}}{r^{2}}=\frac{4 g}{r}+D,
$$

em que $D$ é uma constante de integração. A partir das Eqs. (9) e (10) chega-se à expressão

$$
d \varphi=\frac{v R}{r^{2} \sqrt{D+\frac{4 g}{r}-\frac{v^{2} R^{2}}{r^{2}}}} d r
$$

que, quando integrada, resulta em

$$
\cos (\varphi-\alpha)=\frac{(v R)^{2}-2 g r}{r \sqrt{(v R)^{2} D+4 g^{2}}},
$$

em que $\alpha$ é mais uma constante de integração. Se $\alpha=\mathrm{e}$ $r=R$, quando $\varphi=$, o resultado se torna

$$
\cos \varphi=\frac{R}{r} \frac{(v R)^{2}-2 g r}{\left((v R)^{2}-2 g R\right)},
$$

ou ainda:

$$
\frac{1}{r}=\frac{2 g}{(v R)^{2}}\left(1+\left(\frac{v^{2} R}{2 g}-1\right) \cos \varphi\right),
$$

que representa uma cônica.

Fica claro nesse ponto que, de fato, a mecânica newtoniana é capaz de prever um desvio da luz devido à ação de um campo gravitacional. Resta agora mensurar esse desvio. Para tal, os passos de Soldner serão seguidos, com volta ao sistema de coordenadas cartesianas, tomando as seguintes escolhas: da Fig. $3, r=R-r \cos \varphi ; y=r \operatorname{sen} \varphi$ e $r=\sqrt{(R-x)^{2}+y^{2}}$, e a equação da trajetória é dada por

$$
y^{2}=\frac{v^{2} R^{2}}{g} x+\frac{v^{2}\left[v^{2} R^{2}-4 g R\right]}{4 g^{2}} x^{2},
$$

que, como é de se esperar, é uma função do tipo cônica escrita em coordenadas cartesianas, na forma $y^{2}=p x+$ 
$q x^{2}$, em que o parâmetro $q$ estabelece o tipo de trajetória: $q<0$, elipse; $q=0$, parábola; e $q>0$ hipérbole.

Como no atual trabalho, $v^{2} R^{2}>4 g R$, pois $v$ é a velocidade de um raio de luz, a trajetória obtida é hiperbólica. E Soldner conclui, incisivo [16]:

Assim, quando um raio de luz passa por um corpo celestial, em vez de continuar em linha reta ele é forçado pela atração a descrever uma hipérbole, cujo lado côncavo está voltado diretamente para o corpo atraente.

Da Fig. 4 e das propriedades da hipérbole chega-se a

$$
\tan \omega=\frac{2 g}{v \sqrt{\left(v^{2} R^{2}-4 g R\right)}} .
$$

Soldner, então, considera primeiro um raio de luz emergindo da superfície da Terra, obtendo o resultado de milésimos de segundo e conclui que "Como esse valor máximo é bastante inobservável, seria supérfluo ir mais longe". Em seguida mostra o resultado para o caso do Sol. Usando os valores atuais: $G=6,67 \times 10^{-11} \mathrm{~m}^{3} \cdot \mathrm{kg}^{-1} \cdot \mathrm{s}^{-2}$; $M_{\text {Sol }}=1,98892 \times 10^{30} \mathrm{~kg} ; c=2,998 \times 10^{8} \mathrm{~m} . \mathrm{s}^{-1} ; R_{\text {Sol }}=$ $6,955 \times 10^{8} \mathrm{~m}$, chega-se ao resultado $\omega \approx 0,437 "$. Nesse caso, o desvio deve ser igual ao dobro do valor calculado, como mostrado na Fig. 4. Dessa forma, o resultado final é

$$
\delta=2 \omega \approx 0,875 "
$$

O resultado apresentado por Soldner em seu artigo foi $\delta \approx 0,84$ ". Contudo, na sequência admite que o efeito é desprezível: "Se alguém pudesse observar as estrelas fixas muito perto do Sol, então teria que levar isso muito em conta. Mas como isso não é conhecido que aconteça,

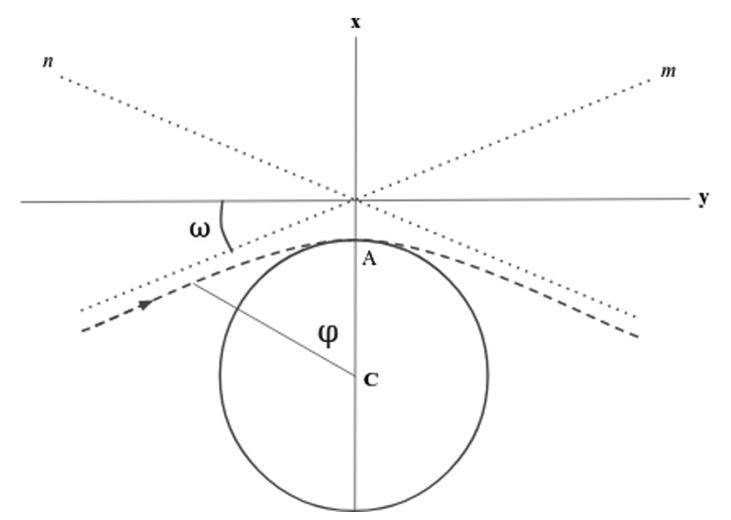

Figura 4: A linha tracejada representa a trajetória de um raio de luz vindo de uma fonte infinitamente distante. O raio de luz segue a trajetória paralela à reta pontilhada $n$, e à medida que se aproxima da fonte gravitacional é desviada, seguindo um trajeto curvilíneo que tangencia a fonte gravitacional no ponto $A$, e segue assintoticamente à reta pontilhada $m$. Importante notar que o desvio total se dá pelo ângulo entre as retas $m$ e $n$, sob as quais o feixe de luz segue assintoticamente, antes e depois da fonte gravitacional. a perturbação causada pelo Sol também pode ser desprezada."

Ao final do artigo, Soldner justifica o fato de considerar um raio de luz como um "corpo pesado" porque acredita que os raios luminosos possuem "as propriedades absolutas [básicas] da matéria". Segundo afirma, "o fenômeno da aberração somente é possível porque os raios luminosos são verdadeiramente materiais". Defende sua pretensão de publicar um trabalho que forneceu resultados que não podem ser observados:

De qualquer forma, não acredito que haja necessidade de me desculpar por haver publicado o presente ensaio somente porque as perturbações não são observáveis. É importante para todos conhecer a teoria, mesmo que não tenha influência notável na prática, tanto quanto estamos interessados no que tem, em retrospecto, real influência. Nosso entendimento seria igualmente aumentado por ambos. Também se pode demonstrar, por exemplo, que a aberração diária, a distorção da rotação da Terra e outras coisas semelhantes são igualmente não observáveis.

Concluindo esta seção, vale destacar que Soldner considerou a velocidade da luz na superfície do corpo massivo, emergindo tangente ao ponto $A$. De fato, com a óptica newtoniana, a velocidade padrão da luz $c$ é sempre definida na fonte emitente. Contudo, a intenção de Soldner seria simular um feixe de luz passando tangente pelo ponto $A$, oriundo de uma fonte infinitamente distante, como mostra a Fig. 4, o que justifica o fator multiplicativo 2, na constante gravitacional, como explica a nota de rodapé 5 . Contudo, isso conduz Soldner a um equívoco, já que, de acordo com a óptica e a mecânica newtonianas, a velocidade do feixe não seria $c$ no ponto $A$. De fato, quando são impostas as condições iniciais, a partir da Eq. (14), Soldner nada comenta sobre a velocidade do raio de luz em $\varphi=0$. No entanto, na sequência, quando se toma o valor $c$ para a velocidade da luz no cálculo da deflexão, fica claro que corresponde à condição imposta da velocidade no ponto $A$. Essa discussão será retomada ao se tratar, na seção 5, do cálculo de Cavendish.

\section{Henry Cavendish: o cálculo correto da deflexão}

O envolvimento de Cavendish, retratado na Fig. 5, na publicação do artigo de Michell de 1784 e a constante troca de correspondência entre si o levaram a investigar outra maneira de medir o efeito da gravidade sobre a luz que se refere à mudança na direção da luz em vez de sua velocidade. Na opinião de McCormmach [12]: "Sem dúvida, ele começou esse estudo óptico-dinâmico como resultado de seu envolvimento com o trabalho de Michell". Cavendish deve ter se convencido de que a retardação gravitacional da luz proposta por Michell seria difícil de detectar e calculou "a curvatura de um raio de luz 


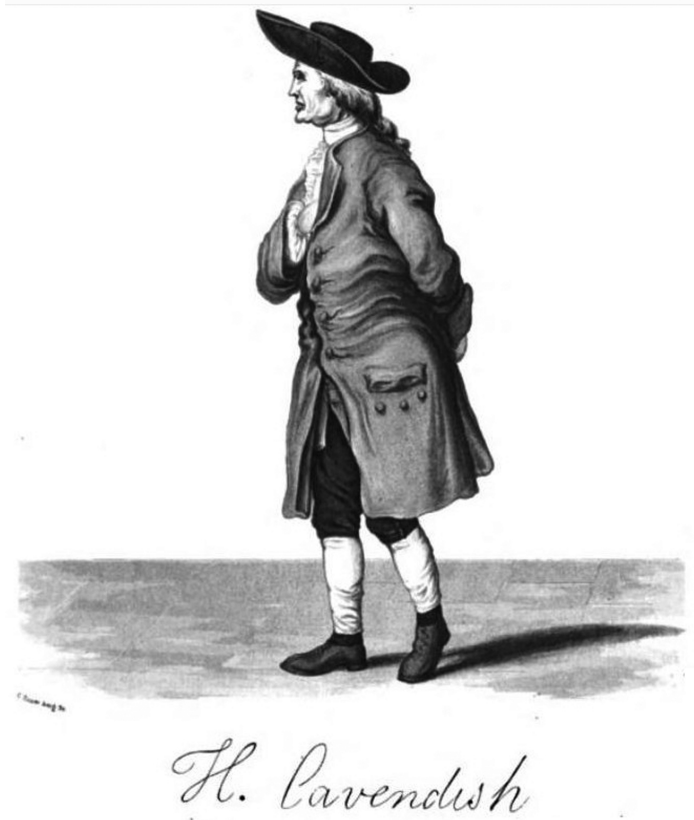

Figura 5: Retrato e assinatura de Henry Cavendish. (Domínio público.)

que passa perto da superfície de qualquer corpo devido à atração desse corpo" [19].

Cavendish nunca publicou seu cálculo da deflexão da luz. Assim, a primeira referência conhecida ao fenômeno do desvio da luz em um campo gravitacional encontra-se numa breve menção encontrada em seus papéis durante o processo de compilação e publicação de sua obra $\sqrt{6}^{7}$ Frank Dyson, um dos responsáveis pela compilação da obra de Cavendish, anotou em 1921: "um rascunho único sobre a curvatura de um raio de luz devido à gravitação, o que é de interesse, pois [essa] possibilidade ... está atualmente atraindo atenção, embora Cavendish estivesse trabalhando dentro de uma teoria corpuscular" [20].

O texto é enigmático $[14,19]$ :

"Para encontrar a deflexão de um raio de luz que passe próximo à superfície de um corpo pela atração desse corpo.

Seja $s$ o centro do corpo e $a$ um ponto na superfície. Se a velocidade do corpo girando em um círculo à distância as do corpo estiver em proporção 1: $u$ para a velocidade da luz,

${ }^{6}$ Os papéis de Cavendish sobre eletricidade foram compilados por James Clerk Maxwell, seu sucessor na cátedra de Cambridge, em 1879. A compilação dos papéis sobre dinâmica, química e astronomia só foi iniciada no começo na Primeira Guerra Mundial e finalizada em 1920 por Frank Dyson, o astrônomo-real, que participou ativamente no esforço de enviar equipes de astrônomos para observação do eclipse de 1919, assim como na análise e divulgação dos resultados, que comprovaram a deflexão da luz e o valor obtido pela teoria da relatividade geral.

${ }^{7}$ A data em que o cálculo foi feito é desconhecida. A marca d'água na folha diz "1802". É bastante provável que tenha sido inspirada pelo artigo de Michell, mas também pode ter saído de seu estudo da órbita dos cometas, já que o manuscrito está dentro de um pacote de documentos sobre esse assunto [22]. então o seno da metade da deflexão do raio será igual a $1 /\left(1+u^{2}\right)$."

Na ausência de detalhes do cálculo pelo próprio Cavendish, o estudo, a seguir, da deflexão baseado na teoria de gravitação newtoniana será apresentado para obter o resultado acima e compará-lo com o resultado de Johann von Soldner, seguindo a solução dada no trabalho de Clifford Will [21].

O problema do movimento da partícula sob ação de uma força central do tipo $F(u)$ em que $u=1 / r$ foi tratado de forma geral por Jacques Binet, que deduziu uma equação que permite encontrar em coordenadas polares o movimento orbital para uma dada lei de força e que usualmente envolve uma equação diferencial ordinária não linear de segunda ordem. A equação de Binet pode ser escrita com 8

$$
F\left(u^{-1}\right)=-m h^{2} u^{2}\left(\frac{d^{2} u}{d \varphi^{2}}+u\right),
$$

em que $h=L / m=r^{2}(d \varphi / d t)$, e $L$ o momento angular que é conservado.

Segundo a óptica newtoniana, partículas de luz são atraídas pela gravidade como as demais partículas. O princípio de equivalência (massa inercial = massa gravitacional) estabelece que a aceleração de um corpo, na presença da gravidade, independe da massa do corpo, de forma que uma partícula de luz sujeita a uma força $F(u) / m=-G M u^{2}$ satisfaz a equação

$$
\frac{d^{2} u}{d \varphi^{2}}+u=\frac{G M}{h^{2}}
$$

A solução da equação é conhecida e dada por 9 [21]

$$
\begin{gathered}
u \equiv \frac{1}{r}=\frac{1}{R(1+e)}(e \cos \varphi+1) \\
r^{2}\left(\frac{d \varphi}{d t}\right)=[\operatorname{GMR}(1+e)]^{\frac{1}{2}},
\end{gathered}
$$

em que $e$ é a excentricidade da órbita e $R$ é a distância do ponto de aproximação mais próxima do centro de força. Como a velocidade da luz é muito grande, sua órbita em um campo fraco, como o do Sol ou de um planeta, será aberta. Das Eqs. (20) e (21), a velocidade da partícula de luz é obtida como

$$
v^{2}=\frac{G M}{R(e+1)}\left(1+2 e \cos \varphi+e^{2}\right) .
$$

No limite, $r \rightarrow \infty, \varphi \rightarrow \frac{\pi}{2}+\omega$, como se observa na Fig. 4 , resultando em

$$
\operatorname{sen} \omega=\frac{1}{e}
$$

\footnotetext{
8 A dedução pode ser encontrada no verbete Binet equation da Wikipedia.

9 Detalhes do cálculo podem ser encontrados em livros de mecânica clássica, como na seção 3.5 da Ref. [23].
} 
O resultado de Cavendish é obtido caso a condição seja de que a velocidade da luz é igual a $c$ no infinito, que satisfaz a concepção newtoniana de que a velocidade da luz deve ser tomada relativamente à fonte emissora onde estiver. Usando essa premissa, chega-se, da Eq. (22), a

$$
c^{2}=\left.v^{2}\right|_{r \rightarrow \infty}=\frac{G M(e-1)}{R},
$$

de modo que

$$
e=1+\frac{1}{\varepsilon}
$$

com $\varepsilon=G M / R c^{2}$. E a metade do ângulo de deflexão é dada por

$$
\operatorname{sen} \omega=\frac{\varepsilon}{1+\varepsilon}
$$

Esse é precisamente o resultado de Cavendish posto em seu manuscrito, já que a distância $a s \equiv \overline{A C} \equiv R$ e a velocidade da órbita circular $v=(G M / R)^{1 / 2}$ e o símbolo de Cavendish $u=\left(\frac{R c^{2}}{G M}\right)^{1 / 2}=\varepsilon^{-1 / 2}$ e chega-se ao mesmo resultado dado na Eq. (24).

Por outro lado, Soldner considerou de forma equivocada a velocidade padrão da luz no ponto $A$ da Fig. 4, tal que a condição a ser imposta é

$$
c^{2}=\left.v^{2}\right|_{\varphi=0}=\frac{G M(1+e)}{R},
$$

e a metade do ângulo de deflexão, dada por Soldner, é

$$
\operatorname{sen} \omega=\frac{\varepsilon}{1-\varepsilon} \text {. }
$$

Apesar das diferenças, os resultados de ambos para a deflexão gravitacional do raio de luz por um corpo massivo podem ser aproximados como

$$
\delta \equiv 2 \omega \approx 2 \frac{G M}{R c^{2}},
$$

que dá 0,875 " para o desvio para o Sol. Esse resultado ficou conhecido como o valor newtoniano para a deflexão.

\section{Einstein e o final da jornada}

No que segue, a trajetória de Einstein, cuja foto ainda jovem, é mostrada na Fig. 6, no caminho da formulação da relatividade geral será abordada, culminando com o cálculo correto para a deflexão da luz, cujo valor coincide com o obtido pelas expedições britânicas em 1919.

\section{1. "O dia mais feliz da minha vida" (1907)}

Em palestra em Kyoto em 14 de dezembro de 1922, Einstein traça a trajetória que o levou a generalizar a sua teoria da relatividade de 1905 (restrita a sistemas inerciais) para incluir sistemas acelerados. Assim se manifestou [24]:

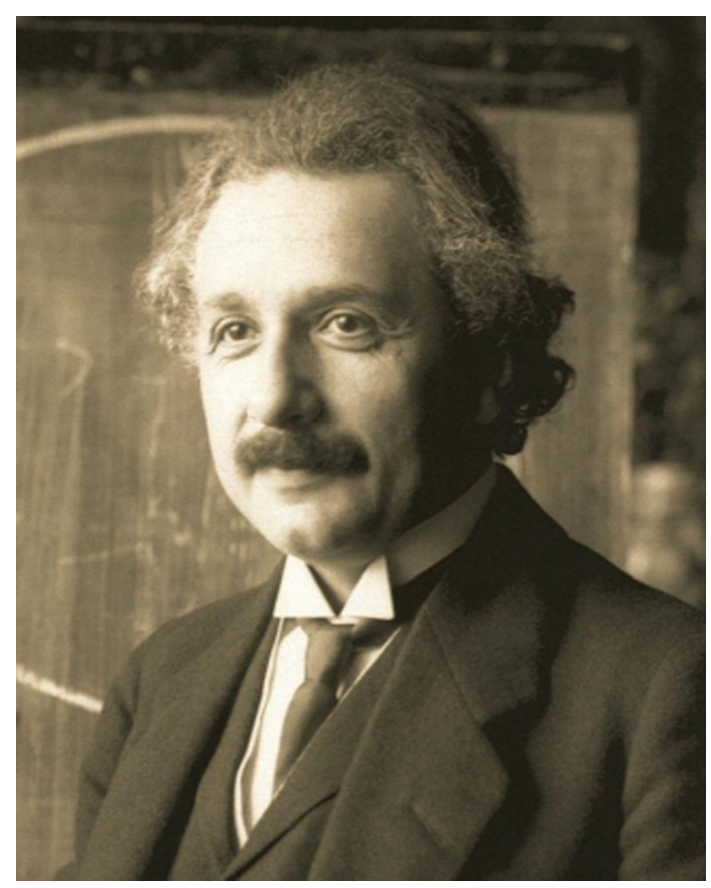

Figura 6: Albert Einstein em 1921. Fonte: Wikipedia.

Em 1907, Johannes Stark me pediu para escrever um texto sobre a teoria da relatividade restrita na revista Jahrbuch der Radioaktivitat [Anuário de Radioatividade]. Enquanto eu estava escrevendo isso, entendi que todas as leis naturais exceto a lei da gravidade podiam ser discutidas dentro do contexto da teoria da relatividade restrita. [...] O ponto mais insatisfatório era o seguinte: apesar de a relação entre inércia e energia ser explicitamente dada na teoria da relatividade restrita $\left[E=m c^{2}\right]$, a relação entre inércia e peso, ou a energia do campo gravitacional, não era claramente elucidada.

E surge o insight de uma mente preparada:

Eu estava sentado na cadeira de meu escritório de patentes em Berna. De repente, um pensamento me assaltou: se alguém cai livremente, não sente o próprio peso. Fiquei chocado. Esse experimento mental simples causou profunda impressão em mim. Isso me levou à teoria da gravitação. Continuei meu pensamento: um homem em queda é acelerado. O que ele sente e julga está acontecendo no referencial acelerado. Decidi estender a teoria da relatividade para o referencial com aceleração. Senti que ao fazer isso eu estaria resolvendo o problema da gravidade ao mesmo tempo. Um homem em queda não sente o próprio peso porque em seu referencial há um novo campo gravitacional que cancela o campo gravitacional devido à Terra. 
No referencial acelerado, precisamos de um novo campo gravitacional.

O artigo de 1907 do Jahrbuch der Radioaktivitat é um documento extraordinário porque mostra os passos que Einstein empreendeu para generalizar a sua teoria da relatividade [25].

\subsection{O experimento do pensamento (Gedanken) e a origem do princípio da equivalência}

Em artigo não publicado de 1919, Einstein antecipara da palestra de Kyoto a ideia básica que o levou a estabelecer o princípio da equivalência [26]. Como usual em seus artigos, Einstein é bastante didático:

Por exemplo, o campo gravitacional tem uma existência apenas relativa, de maneira semelhante ao campo elétrico gerado por indução eletromagnética. Para um observador em queda livre do telhado de casa não existe, durante a queda, ao menos na vizinhança imediata, nenhum campo gravitacional. Ou seja, se o observador soltar algum objeto, aquilo permanecerá, em relação a ele, em repouso ou movimento uniforme, independente de sua natureza química ou física (é claro que essa consideração ignora a resistência do ar). O observador está certo, portanto, em interpretar seu estado como sendo de "repouso". A experiência estranha e confirmada de que todos os corpos no mesmo campo gravitacional caem com a mesma aceleração imediatamente ganha, através dessa ideia, um sentido físico profundo. Porque se apenas uma coisa caísse no campo gravitacional de maneira diferente das outras, um observador poderia reconhecer que está em um campo gravitacional e está caindo. Mas se tal coisa não existe - a experiência mostra isso com alta precisão - então não há razão objetiva para um observador se considerar caindo em um campo gravitacional. Ao contrário, ele tem todo o direito de se considerar em repouso e sua vizinhança livre de campos, em termos gravitacionais.

Einstein faz a conjectura:

Se soubermos as leis da natureza em um sistema K (livre de gravidade), então poderemos por mera transformação saber as leis no sistema K', i.e., aprendermos sobre as propriedades físicas de um campo gravitacional por simples especulação. O que temos aqui é a hipótese de que o princípio de relatividade também vale em sistemas de coordenadas que estejam mutuamente acelerados entre si, e que todas as propriedades físicas do espaço que valem em K' são completamente equivalentes ao campo gravitacional (hipótese de equivalência).
Após ressaltar que a hipótese da equivalência é apoiada pela experiência de Eötvös, Einstein prossegue em termos bastante simples. A lei do movimento de Newton estabelece que

$$
m_{I} a=F_{G}
$$

em que $m_{I}$ é a massa inercial, responsável pela reação inercial do corpo, $a$ é a aceleração de queda, e $F_{G}$ é a força gravitacional exercida pela Terra. Por outro lado,

$$
F_{G}=m_{G} g,
$$

em que $g$ é a intensidade do campo gravitacional e $m_{G}$ é a massa gravitacional, constante responsável pela influência do campo gravitacional sobre o mesmo corpo. As constantes $m_{I}$ e $m_{G}$ são, por definição, completamente independentes. Das duas equações, obtém-se

$$
m_{I} a=m_{G} g .
$$

Para concordar com a lei válida experimentalmente, então "deve ser verdade" que

$$
a=g,
$$

e conclui:

Portanto, o fato experimental de mesma queda para todos os corpos pode, no espírito da mecânica newtoniana, ser vista como a igualdade das massas gravitacional e inercial, que do ponto de vista da mecânica newtoniana não é de modo algum autoevidente. Este teorema tem sido confirmado com precisão extraordinária pelos testes de Eötvös.

Arthur Miller, em sua interessante discussão sobre o experimento de pensamento do "observador em queda livre" de Einstein faz uma conjectura perfeitamente razoável de como Einstein poderia ter mostrado, além da equivalência das massas inercial e gravitacional, por que o observador "teria o direito de se considerar em um estado de repouso". O argumento merece ser reproduzido aqui.

Sejam $K^{\prime}$ o sistema de referência associado ao observador em queda livre e $K$ o sistema de referência de um observador em repouso no chão. Os eixos $y$ na vertical estão alinhados. Considere que as velocidades sejam suficientemente pequenas para que a mecânica newtoniana possa ser usada. A equação do movimento relativa a $K$ do homem em queda livre é

$$
m_{T} \frac{d^{2} y}{d t^{2}}=-m_{G} g
$$

em que $m_{T}\left(m_{G}\right)$ é a massa inercial (gravitacional) do observador em queda e $g$ a aceleração 
do observador devida ao campo gravitacional. Supondo que todas as velocidades são pequenas de modo que a relatividade restrita possa ser desprezada, a transformação entre $K$ e $K^{\prime}$ é dada por

$$
y^{\prime}=y-\frac{a t^{2}}{2},
$$

em que $a$ é a aceleração constante do corpo em relação a $K, t^{\prime}=t$ e $y^{\prime}(y)$ refere-se a $K\left(K^{\prime}\right)$. A segunda lei de Newton em $K^{\prime}$ é, então, dada por

$$
m_{T} \frac{d^{2} y^{\prime}}{d t^{\prime 2}}+m_{T} a=-m_{G} g .
$$

O passo inicial é assumir que $m_{T}=m_{G}=m$. O passo seguinte é supor que o observador em queda livre em cuja vizinhança "não existe campo gravitacional" tem o direito de interpretar seu estado como repouso. Isso exige que $a=-g$ e, portanto,

$$
m \frac{d y^{\prime 2}}{d t^{\prime 2}}=0 .
$$

A conclusão é que o homem caindo está em estado de repouso relativamente ao observador em $K^{\prime}$, apesar de estarem ambos em queda livre.

Na seção "Princípio da relatividade e gravitação" do artigo de revisão de 1907, Einstein apresenta uma teoria dependente de coordenadas, usando seu princípio da equivalência (Aequivalenzprinzip) que estabelece que sistemas em repouso na presença de um campo gravitacional são equivalentes a sistemas acelerados com $g$ :

Assumiremos, portanto, uma equivalência física completa entre um campo gravitacional e uma aceleração correspondente de um sistema referencial. Essa suposição estende o princípio da relatividade ao movimento de translação uniformemente acelerado de um sistema referencial.

Einstein trata os sistemas físicos de referência com varetas de medição e leituras de relógios, de maneira semelhante ao artigo da relatividade restrita de 1905, e chega a novos resultados sem introduzir matemática complicada na teoria.

Determina a relação entre o tempo $\tau$ no sistema uniformemente acelerado e o tempo local $\sigma$ de um evento pontual, dada por

$$
\sigma=\tau\left(1+\frac{\Phi}{c^{2}}\right),
$$

em que $\Phi$ é o potencial gravitacional. Portanto, a dilatação temporal se estende para o caso gravitacional:
Assim, para um observador situado em algum lugar do espaço, o relógio no ponto $\mathrm{P}$ corre $\left(1+\Phi / c^{2}\right)$ vezes mais rápido que o relógio na origem do sistema de coordenadas. Nesse sentido poderíamos dizer que os processos que ocorrem no relógio e, de modo mais geral, qualquer processo físico, evolui mais rapidamente, quanto maior o potencial gravitacional na posição onde ocorre o processo.

A seguir, estabelece o efeito Doppler gravitacional, ou seja, deslocamento das raias espectrais (red shift) devido à gravidade:

Pode ser concluído, que o comprimento de onda da luz vindo da superfície do Sol, originária de um tal produtor (de linhas espectrais) é maior do que o da luz produzida pela mesma substância na superfície da Terra de uma parte em dois milhões.

Ao analisar o efeito da gravitação sobre fenômenos eletromagnéticos, Einstein mostra que a velocidade da luz, postulada como constante em sistemas de referência inerciais, depende do potencial gravitacional de uma forma similar dada por

$$
c=c_{0}\left(1+\frac{\Phi}{c^{2}}\right) .
$$

E conclui que "disso segue que os raios de luz que não se propagam ao longo do eixo são defletidos pelo campo gravitacional. Pode ser facilmente verificado que a mudança na direção é de $\left(a / c^{2}\right) \operatorname{sen} \theta$ por $\mathrm{cm}$ de trajetória da luz, na qual $\theta$ representa o ângulo entre a direção da gravidade e a do raio de luz."

Einstein, já em 1907, faz a predição do encurvamento dos raios luminosos. Mas ressalta que "Infelizmente o efeito do campo gravitacional terrestre é tão pequeno, de acordo com a nossa teoria, que não há previsões para comparar essa teoria com a experiência."

Einstein ainda assegura, na conclusão do artigo, que a equivalência massa-energia é preservada em sistemas acelerados: "Assim, a proposição derivada, na qual a uma quantidade de energia $E$ corresponde uma massa $E / c^{2}$ é válida não apenas para a massa inercial, mas também para a massa gravitacional, se a suposição introduzida (princípio da equivalência) for correta."

\subsection{O artigo de 1911}

Quatro anos após a introdução das ideias para estender a teoria da relatividade restrita para incluir a gravitação, em junho de 1911, durante sua permanência na Universidade de Praga, Einstein publicou "Sobre a influência da gravitação na propagação da luz" [27].

Essa abordagem foi uma tentativa de corrigir a previsão de 1907, a qual, segundo o próprio Einstein, não o havia deixado satisfeito. Além disso, deve ter lhe ocorrido que o efeito poderia ser observado em um eclipse solar. 
Nessa nova tentativa, Einstein faz uso de dois princípios. O primeiro é o princípio da equivalência, segundo o qual um observador em um referencial uniformemente acelerado equivale a um referencial na presença de um campo gravitacional uniforme [27]:

Chegaremos, porém, a uma interpretação muito satisfatória de tal lei experimental [a queda idêntica de todos os corpos no campo da gravidade], se admitirmos que os sistemas $K$ e $K^{\prime}$ [acelerado] se equivalem completamente do ponto de vista físico.

O segundo é o princípio da relatividade, em que os processos físicos devem ser os mesmos para referenciais equivalentes:

Enquanto nos cingirmos aos fenômenos puramente mecânicos abrangidos pelo domínio de validade da mecânica newtoniana, não oferece dúvida a equivalência dos sistemas $K$ e $K^{\prime}$; mas essa equivalência só atingirá um significado de maior profundidade se a admitirmos para todos os fenômenos físicos, isto é, se as leis da Natureza referidas a $\mathrm{K}$ coincidirem inteiramente com as leis referidas a $K^{\prime}$.

No que segue, os detalhes do artigo de Einstein serão expostos.

\subsubsection{Uma hipótese a respeito da natureza física do campo gravitacional}

Considere dois referenciais, $K$ e $K^{\prime}$. No referencial $K$, as coordenadas são $x, y, z$ e há um campo gravitacional de aceleração $g$ orientado no sentido de $z$ decrescente. No referencial $K^{\prime}$ não existe campo de gravitação; porém, esse referencial se move com aceleração uniforme $g$ no sentido de $z$ crescente:

As equações de movimento de um ponto material que não está sujeito a ação de nenhum outro ponto material são dadas, em cada um dos referenciais, por

$$
\begin{gathered}
\frac{d^{2} \underline{r}}{d t^{2}}=\left(\frac{d^{2} x}{d t^{2}}, \frac{d^{2} y}{d t^{2}}, \frac{d^{2} z}{d t^{2}}\right)=(0,0,-g), \text { em } K \\
\frac{d^{2} \underline{r}^{\prime}}{d t^{2}}=\left(\frac{d^{2} x^{\prime}}{d t^{2}}, \frac{d^{2} y^{\prime}}{d t^{2}}, \frac{d^{2} z^{\prime}}{d t^{2}}\right)=(0,0,-g), e m K^{\prime} .
\end{gathered}
$$

No referencial $K$, as equações seguem da segunda lei de Newton e do princípio de equivalência fraco, que estabelece que as massas inercial e gravitacional de um corpo são equivalentes. Em $K^{\prime}$ a equação segue do princípio de relatividade de Galileu.

A partir da análise do movimento da partícula, é impossível se distinguir entre a existência de um campo gravitacional e um referencial uniformemente acelerado.

\subsubsection{Sobre a gravitação da energia}

Um resultado obtido na teoria da relatividade restrita é que a massa inercial de um corpo cresce com a energia que ele contém. Se o aumento da energia da partícula é $E$, então sua massa inercial crescerá de $E / c^{2}$, sendo $c$ a velocidade da luz.

Sejam dois observadores $S_{1}$ e $S_{2}$ equipados com sistemas de medição e separados entre si por uma distância $h$ ao longo do eixo $z$. Seja $E_{2}$ uma quantidade de energia emitida em forma de radiação de $S_{2}$ em direção a $S_{1}$. Para analisar o que ocorre no sistema $K^{\prime}$, considere-se que, no instante em que $S_{2}$ emite a radiação em direção a $S_{1}$, o sistema $K^{\prime}$ tem velocidade nula em relação ao referencial $K_{0}$, que é um referencial livre de aceleração no espaço livre (ver Fig. 7). A radiação atinge $S_{1}$ após um intervalo (em primeira ordem de aproximação) dado por

$$
\Delta t \approx \frac{\Delta z}{v}=\frac{h}{c} .
$$

Ao atingir o observador $S_{1}$, o mesmo terá se deslocado em direção à fonte e terá adquirido uma velocidade

$$
v=a t=g h / c .
$$

Pela teoria da relatividade, a radiação que chega em $S_{1}$ não terá energia $E_{2}$, mas sim uma energia maior, $E_{1}$, dada em primeira ordem de aproximaçã ${ }^{10}$ como

$$
E_{1}=E_{2}(1+v / c)
$$

Substituindo-se a velocidade em termos de $g, h$ e $c$, chegase a

$$
E_{1}=E_{2}(1+g h / c)
$$

Ao se assumir que no referencial $K$ (que não é acelerado, mas tem um campo de gravitação) a física deve ser equivalente, então a energia em $S_{1}$ deve ser dada por

$$
E_{1}=E_{2}(1+g h / c)=E_{2}+E_{2} \Phi / c^{2},
$$

em que $\Phi$ é o potencial gravitacional de $S_{2}$ em relação a $S_{1}$.

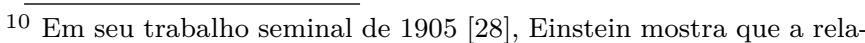
ção entre as energias de um raio de luz medidas em um referencial estacionário, $E$, e aquela medida em um referencial em movimento, $E$ ', é dada por

$$
E^{\prime} / E=\sqrt{[(1-v / c) /(1+v / c)]} .
$$

A expansão em série de Taylor da função $f(x) \equiv$ $\sqrt{[(1-x) /(1+x)]}$ em primeira ordem em torno de $x=0$ nos fornece:

$$
f(x) \approx 1-x+O\left(x^{2}\right) .
$$

No caso em questão, para velocidades muito abaixo da velocidade da luz, $E^{\prime} / E \approx 1-v / c$. No trabalho de Einstein, a radiação encontra-se propagando-se no mesmo sentido da velocidade de translação. No presente caso, a radiação propaga-se no sentido contrário; assim, ajustamos a relação acima, trocando o sinal da velocidade:

$$
E^{\prime} / E \approx 1+v / c
$$



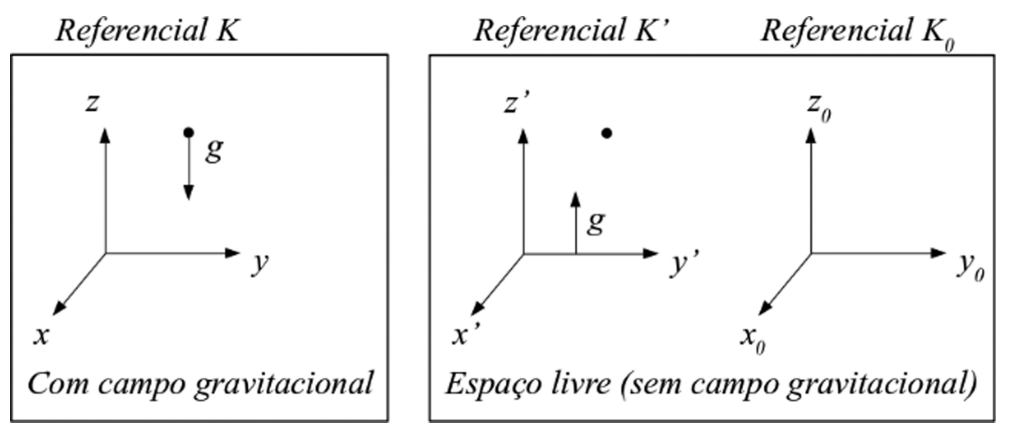

Figura 7: Representação dos referenciais $K$, na presença de um campo de gravitação; $K^{\prime}$, como um referencial acelerado no espaço livre; e $K_{0}$, como um referencial estático no espaço livre.

\subsubsection{Tempo e velocidade da luz no campo gravitacional}

No trabalho de Einstein de 1905, uma relação análoga à da energia é obtida para as frequências da luz observadas nos referenciais estático e em movimento relativo. Dessa forma, em primeira ordem de aproximação (já ajustando o sinal da velocidade):

$$
\nu^{\prime} / \nu=\sqrt{\frac{1+v / c}{1-v / c}} \approx 1+v / c .
$$

Assim, ao se denotar por $\nu_{2}$ a frequência da radiação emitida em $S_{2}$ e $\nu_{1}$, a frequência medida em $S_{1}$, por argumentos análogos ao caso da energia, será medida no referencial $K^{\prime}$ :

$$
\nu_{1}=\nu_{2}(1+g h / c)
$$

que é uma relação que nos remonta ao efeito Doppler. No caso do referencial $K$, vê-se que esse efeito representa o efeito Doppler gravitacional, o qual se reescreve como:

$$
\nu_{1}=\nu_{2}\left(1+\Phi / c^{2}\right)
$$

Ao se considerar $i$ ) que a frequência está associada ao número de períodos por unidade de tempo e $i i$ ) que o número de períodos, $n$, é o mesmo nas duas posições consideradas, conclui-se que essa diferença na frequência ocorre porque o tempo não se passa da mesma forma nos dois pontos $S_{1}$ e $S_{2}$. Como uma consequência dessa relação, a velocidade de propagação da luz deveria ser diferente nos dois pontos. Se $c$ é a velocidade da luz no ponto $S_{2}$ e $c_{0}$ é a velocidade da luz em $S_{1}$, entãd

$$
c=c_{0}\left(1-\Phi / c^{2}\right) \text {. }
$$

Essa noção de que a velocidade da luz deveria mudar na presença de campo gravitacional é revisada na proposição da teoria da relatividade geral, em que a velocidade da luz é considerada como $c$, mesmo na presença de gravitação.

\subsubsection{Desvio de raios de luz no campo gravitacional}

A mudança da velocidade da luz na presença de campo gravitacional é análoga à mudança da velocidade da luz quando se muda o meio de propagação. Assim, o uso do princípio de Huygens para a propagação da luz na presença de gravitação deve prever a deflexão dos raios de luz.

Considerem-se dois pontos $P_{1}$ e $P_{2}$ separados por uma distância infinitesimal $\Delta r$ como mostrado na Fig. 8. Ligando-se os dois pontos, há uma frente de onda se propagando. Sem a presença do campo gravitacional, a frente de onda propaga-se sem alterar sua direção. Na presença do campo, a diferença de velocidade é responsável por alterar a direção da frente de onda. O ângulo de deflexão pode ser obtido a partir dos caminhos percorridos pelas frentes de onda emitidas em $P_{1}$ e $P_{2}$, divididos pela distância de separação entre os mesmos, e o resultado é

$$
d \theta=\frac{\left(c_{1}-c_{2}\right) d t}{\Delta r}
$$

\footnotetext{
11 Essa a aproximação sugerida por Einstein. Da relação entre as frequências, deduzimos que $\Delta t_{1}$ e $\Delta t_{2}$ estão relacionados por meio de $\Delta t_{1}=\Delta t_{2} /\left(1+\Phi / c^{2}\right) \approx \Delta t_{2}\left(1-\Phi / c^{2}\right)$. Pela regra da cadeia a relação para a velocidade da luz é inferida.
}
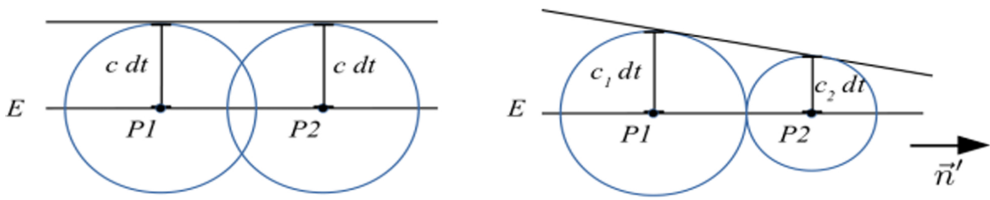

Figura 8: Representação das frentes de onda em um espaço livre e na presença de campo gravitacional. 
Se $\Delta r$ for entendido como sendo a componente de $\Delta \vec{r}=$ $\overrightarrow{r_{2}}-\overrightarrow{r_{1}}$ na direção de $\vec{n}^{\prime}$, que é a direção perpendicular à frente de onda, então, no limite $\Delta r \rightarrow 0$, pode-se escrever

$$
\begin{aligned}
\lim _{\Delta r \rightarrow 0} \frac{\left(c_{1}-c_{2}\right)}{\Delta r} & =\lim _{\Delta r \rightarrow 0} \frac{d c}{\Delta r}=\lim _{\Delta r \rightarrow 0} \frac{d c}{d \vec{r}} \cdot \vec{n}^{\prime} \\
& =\nabla c \cdot \vec{n}^{\prime}=\frac{\partial c}{\partial n^{\prime}},
\end{aligned}
$$

e, portanto,

$$
d \theta=\frac{\partial c}{\partial n^{\prime}} d t
$$

O diferencial $d t$ pode ser expresso em termos do comprimento percorrido pela luz, $d s$, no intervalo $d t$, i.e. $d t=d s / c$; a derivada direcional de $c$ ao longo da direção normal à frente de onda pode ser expressa como

$$
\frac{\partial c}{\partial n^{\prime}}=\nabla c \cdot \vec{n}^{\prime}=\frac{1}{c} \nabla \Phi \cdot \vec{n}^{\prime}=\frac{1}{c} \frac{\partial \Phi}{\partial n^{\prime}} .
$$

Dessa forma, o ângulo total de deflexão deve ser dado pela integração dos diferenciais angulares, levando a

$$
\alpha=\int \frac{1}{c^{2}} \frac{\partial \Phi}{\partial n^{\prime}} d s
$$

Considerando então o caso em que o raio de luz passa próximo a um corpo bastante massivo, descrito por um potencial newtoniano, tem-se que

$$
\frac{\partial \Phi}{\partial n^{\prime}}=\nabla \Phi \cdot \vec{n}^{\prime}=-\frac{G M}{r^{2}} \hat{r} \cdot \vec{n}^{\prime}=-\frac{G M}{r^{2}} \cos \varphi .
$$

A Fig. 9 representa geometricamente o fenômeno.

Note-se que o comprimento percorrido pela luz possui elemento de comprimento dado por

$$
d s=\Delta d(\cot (\varphi-\pi / 2))=-\Delta \frac{d \varphi}{\cos ^{2} \varphi},
$$

em que $\Delta$ é o raio da estrela. Percebe-se ainda que a distância $r$ que localiza a frente de onda pode ser expressa como

$$
r=\frac{\Delta}{\operatorname{sen}(\varphi-\pi / 2)}=\frac{\Delta}{\cos \varphi} .
$$

Assim, a deflexão é dada por

$$
\begin{aligned}
\alpha & =\int_{-\pi / 2}^{\pi / 2} \frac{1}{c^{2}} \frac{G M}{\left(\frac{\Delta}{\cos \varphi}\right)^{2}} \cos \varphi \frac{\Delta}{\cos ^{2} \varphi} d \varphi \\
& =\int_{-\pi / 2}^{\pi / 2} \frac{1}{c^{2}} \frac{G M}{\Delta} \cos \varphi d \varphi=\frac{2}{c^{2}} \frac{G M}{\Delta} .
\end{aligned}
$$

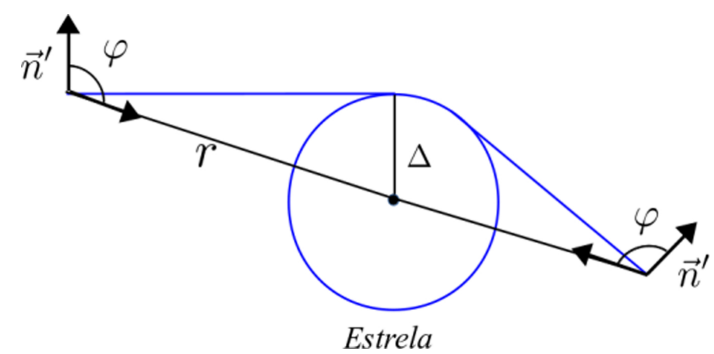

Figura 9: Raio de luz sendo defletido por uma estrela de raio $\Delta$
Usando-se os valores para o caso do Sol, Einstein apresentou o resultado:

Um raio de luz que passasse junto do Sol sofreria assim uma deflexão de $4 \cdot 10^{-6}=0,83$ segundos de arco.

E, ao final do artigo, conclama os astrônomos a procurar observar o efeito:

Seria de extrema conveniência que os astrônomos se ocupassem da questão que aqui foi esboçada, ainda que ela se apresente insuficientemente fundamentada com os raciocínios anteriores, ou até inteiramente aventurosa. Porque, independentemente de qualquer teoria, levanta-se a questão de saber se os meios de que atualmente se dispõe são capazes de registrar uma influência dos campos de gravidade sobre a propagação da luz.

É possível que nem Einstein ${ }^{12}$ nem os leitores do $A n$ nalen der Physik tenham imaginado que 110 anos antes quase exatamente o mesmo valor 0,84 " tinha sido calculado previamente. $\mathrm{O}$ artigo foi recebido com a mesma indiferença que o de Soldner.

No entanto, Einstein buscou o apoio da comunidade astronômica, por intermédio do físico Leo Pollack da Universidade de Praga, que, em agosto de 1911, informou o Observatório de Berlim acerca da predição do encurvamento gravitacional da luz. Em seguida, Einstein logo escreveu para Erwin Freundlich, assistente no Observatório de Berlim, iniciando uma duradoura colaboração científica.

Já em 1912, uma expedição argentina, liderada por Charles Perrine, do Observatório de Córdoba, chegou ao Brasil para observar o eclipse solar [29]. Perrine conhecia o interesse de Freundlich na medida da deflexão da luz. Uma expedição inglesa, liderada por Arthur Eddington, também veio ao Brasil. Nada foi observado por causa das chuvas.

\subsection{Em busca da teoria da relatividade geral}

Como visto, o caminho na direção da teoria da relatividade geral foi um processo longo, que, grosso modo, se iniciou logo após a apresentação da relatividade restrita em 1905. Já em 1907, Einstein começa a se deparar com questões relacionadas ao princípio da relatividade e sua

\footnotetext{
12 No entanto, o historiador Jean Eisenstaedt [14] encontrou um indício de que Einstein estaria ciente do encurvamento da luz no contexto newtoniano. Em carta do verão de 1913, Einstein agradece o empenho do astrônomo Erwin Freundlich na questão do encurvamento dos raios de luz e observa: "que a ideia da curvatura dos raios de luz tenha aparecido nos tempos da teoria da emissão é bastante natural assim como o resultado numérico exato é consistente com a hipótese de equivalência". Eisenstaedt especula que ele tenha obtido essa informação do livro de divulgação de Aaron David Bernstein (Popular Books on Natural Science de 1869) que Einstein leu "com absorvente atenção" como confessou em suas Notas Autobiográficas.
} 
incompatibilidade com a teoria newtoniana, a qual previa uma propagação instantânea para a força gravitacional. O resultado das reflexões oriundas desses questionamentos culmina na proposição do princípio da equivalência, que pode ser entendido como um dos pontos seminais da teoria da relatividade geral. Nos anos seguintes, Einstein aprofunda-se nos estudos das implicações do princípio da relatividade para a gravitação.

Após deixar Praga em 1911, Einstein assumiu uma posição na Escola Politécnica de Zurique, iniciando colaboração com seu antigo colega, o matemático Marcel Grossmann, com o objetivo de generalizar o cálculo vetorial quadridimensional desenvolvido por Minkowski, Sommerfeld, Laue e outros. Grossmann logo encontrou nas formas de cálculo diferencial de Riemann, Christoffel, Ricci e Levi-Civita as ferramentas matemáticas para formular uma teoria covariante para o campo gravitacional. Einstein e Grossmann chegaram a construir uma equação de campo que satisfazia as exigências de conservação de energia e que reproduzia a equação de Poisson no limite newtoniano. Em 1913, publicam o livreto Esboço de uma teoria da relatividade generalizada e de uma teoria da gravitação (Einstein e Grossmann 1913) ${ }^{13}$, dividida em duas partes: a primeira com uma base física desenvolvida por Einstein e a segunda com a teoria matemática escrita por Grossmann. O trabalho apresenta o instrumental matemático do cálculo tensorial que permitiu formular uma teoria com equações de movimento covariantes de forma geral, cujo conceito fundamental consistia na interpretação do tensor métrico $g_{\mu \nu}$ representando o campo gravito-inercial.

Einstein avaliou, com seu amigo Michele Besso, as consequências da teoria Entwurf por meio do cálculo do avanço do periélio de Mercúrio, mas encontrou um resultado significativamente distante do valor observado. Embora tenha manifestado, nos dois anos seguintes, seu contentamento com a teoria, Einstein considerava ainda a teoria restrita do ponto de vista da covariância. Mas, em artigo posterior, mostrou que essa restrição deveria ser esperada. Em um segundo artigo, Einstein e Grossmann fizeram uma reformulação variacional da teoria, identificando o grupo de covariância das equações de movimento. Já em Berlim, ao se tornar membro da Academia Prussiana de Ciências, Einstein publicou, no verão de 1914, um artigo de revisão da teoria Entwurf intitulado "Base formal da teoria da relatividade geral". O artigo, dividido em cinco seções, antecipa a estrutura do artigo de revisão final de 1916. No final, Einstein discute as predições do encurvamento gravitacional da luz e do desvio para o vermelho e recupera o limite newtoniano. No entanto, faltava o resultado correto para o avanço do periélio de Mercúrio. Um segundo fracasso foi apontado por Levi-Civita em abril de 1915, que mostrou falhas na derivação das equações de campo, minando a confiança de Einstein na sua teoria.

Depois de intensa correspondência, Einstein encontrou outros problemas na teoria Entwurf e em setembro da-

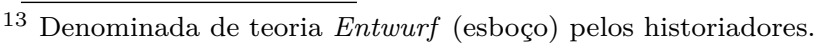

quele ano começou a considerar formas alternativas para as equações de campo gravitacional. Einstein reconsiderou algumas candidatas para as equações de campo que havia desenvolvido em 1912 e concluiu o trabalho em quatro comunicações à Academia Prussiana, nos dias 4, 11, 18 e 25 de novembro, que foram publicadas na Sitzungsberichte. Nas três primeiras, Einstein derivou equações de campo, ainda não definitivas, encontrou o valor correto do avanço do periélio de Mercúrio e, na última, apresentou a forma final das atualmente chamadas "equações de Einstein" da relatividade geral 14

Em março de 1916, apareceu o artigo de revisão final "Os fundamentos da teoria da relatividade geral" [30], que completa a obra seminal de Albert Einstein.

Em 1933, exilado da Alemanha, assim Einstein se manifestou em uma conferência em Glasgow sobre as origens da relatividade geral [32]:

Os anos na escuridão em busca de uma verdade que se percebe, mas que não se consegue exprimir, o intenso desejo e as alternâncias de confiança e perplexidade, até que se consiga esclarecer e compreender, são conhecidos apenas por aquele que os experimenta.

\subsection{A versão final da teoria da relatividade geral: $\mathrm{O}$ artigo de $\mathbf{2 0 1 6}$}

No artigo de 1916, Einstein reúne os elementos essenciais da sua teoria. O trabalho é dividido em cinco partes:

\section{A. Considerações fundamentais sobre o postulado da relatividade}

O trabalho se inicia com a apresentação de algumas considerações a respeito do princípio da relatividade (conforme entendido no contexto da relatividade restrita) e da necessidade de sua generalização: "as leis da física devem ser de tal natureza que elas se aplicam a sistema de referência de qualquer tipo de movimento". E para que isso fosse possível, Einstein estabelece o postulado/princípio da covariância geral:
"As leis gerais da natureza devem ser expres- sas por equações que sejam válidas para todos os sistemas de coordenadas, isto é, que sejam covariantes com respeito a quaisquer substi- tuições que se queiram (covariância geral)".

A implementação desse princípio implica na utilização de novos instrumentos matemáticos que são descritos na segunda parte do artigo.

\section{B. Subsídios matemáticos para a formulação das equações covariantes gerais}

\footnotetext{
14 Deve-se mencionar a competição com David Hilbert que, após a visita de Einstein em que apresentou a teoria Entwurf e, sendo informado dos progressos recentes de Einstein, se antecipou e submeteu sua versão para ser publicada na Nachrichten da Academia de Göttingen em 20 de novembro, cinco dias antes da comunicação final de Einstein. [31, p. 257].
} 
Einstein introduz todo o instrumental matemático para a construção de sua teoria: quadrivetores (covariantes e contravariantes), tensores, convenção da soma 15 $\left(x_{\mu} x^{\mu}=\sum_{\mu} x_{\mu} x^{\mu}\right)$, elemento de linha $\left(d s^{2}\right.$, determinado a partir dos diferenciais das coordenadas), equação de geodésica, tensor métrico $\left(g_{\mu \nu}\right.$ e seu inverso $\left.g^{\mu \nu}\right)$, conexã ${ }^{16}$ $\left(\Gamma_{\mu \nu}^{\rho}=\{\mu \nu \rho\}\right.$, que é determinada a partir do tensor métrico e suas derivadas, como mostrado mais adiante), derivada covariante, tensor de curvatura $\left(B_{\mu \nu \sigma}^{\rho}\right)$, enfim, todos os elementos de geometria diferencial necessários para o desenvolvimento de sua teoria. Nessa seção, o tensor métrico é apresentado como sendo o campo fundamental da teoria. As equações que descrevem a interação gravitacional são apresentadas na terceira parte.

\section{Teoria do campo gravitacional}

A terceira seção inicia-se com as equações de movimento dos pontos materiais em um campo gravitacional. São as equações de geodésicas, que são as curvas de menor distância entre dois pontos do espaço-tempo:

$$
\frac{d^{2} x^{\alpha}}{d s^{2}}+\Gamma_{\mu \nu}^{\alpha} \frac{d x^{\mu}}{d s} \frac{d x^{\nu}}{d s}=0,
$$

em que o elemento de linha $d s$ é determinado a partir de

$$
d s^{2}=g_{\mu \nu} d x^{\mu} d x^{\nu}
$$

e a conexão é dada pelos símbolos de Christoffel

$$
\Gamma_{\mu \nu}^{\rho}=\frac{1}{2} g^{\rho \beta}\left(\partial_{\nu} g_{\beta \mu}+\partial_{\mu} g_{\beta \nu}-\partial_{\beta} g_{\mu \nu}\right),
$$

sendo $\partial_{\nu} g_{\beta \mu}=\frac{\partial g_{\beta \mu}}{\partial x^{\nu}}$.

A partir das equações geodésicas, constata-se que o movimento das partículas é passível de determinação quando o tensor métrico é conhecido. Este, por sua vez, é determinado a partir de outro conjunto de equações construídas a partir de duas hipóteses:

i) as equações devem ser de segunda ordem;

ii) as equações devem ser lineares na segunda derivada.

Essas condições garantem que a nova teoria tenha equações análogas à equação de Newton para gravitação e, em particular, que esta última seja obtida como caso limite da teoria de Einstein. As equações propostas relacionam, de um lado, a geometria do espaço-tempo, manifesta como contrações do tensor de curvatura de Riemann (no caso o tensor de Ricci, que depende da métrica e da primeira e segunda derivadas do tensor métrico), e de outro lado, o conteúdo de "matéria", por meio do tensor energia-momento $\left(T_{\mu \nu}\right)$ e do seu traço $\left(T=g^{\mu \nu} T_{\mu \nu}\right)$ :

$$
\partial_{\rho} \Gamma_{\mu \nu}^{\rho}+\Gamma_{\mu \sigma}^{\rho} \Gamma_{\nu \rho}^{\sigma}=-\kappa\left(T_{\mu \nu}-\frac{1}{2} g_{\mu \nu} T\right) .
$$

Nessa expressão, $\kappa$ é uma constante relacionada à constante da gravitação universal. Einstein explica que por

\footnotetext{
15 A convenção da soma, introduzida por Einstein, estabelece que, quando índices repetidos aparecem em um mesmo termo, há uma soma implícita.

16 Aqui há uma diferença de sinal em comparação com a do artigo original de Einstein. A convenção utilizada aqui é mais moderna e mais compatível com a literatura moderna.
}

"matéria" entende-se tudo aquilo que não seja gravitacional. É interessante ressaltar que a forma mais moderna e conhecida das equações de Einstein é apresentada na forma

$$
R_{\mu \nu}-\frac{1}{2} g_{\mu \nu} R^{\mu \nu}=\frac{8 \pi G}{c^{4}} T_{\mu \nu}
$$

em que $R_{\mu \nu}$ é o tensor de Ricci obtido a partir da contração do tensor de curvatura $\left(R_{\mu \nu}=B_{\mu \nu \rho}^{\rho}\right)$ e $R$ é o escalar de curvatura $\left(R=g^{\mu \nu} R_{\mu \nu}\right)$. Essa forma foi deduzida por Hilbert no contexto do formalismo lagrangeano e, por isso, a lagrangeana de onde essa equação é derivada é chamada de lagrangeana de Einstein-Hilbert. (Ver nota de rodapé 2.)

Após a obtenção das equações de campo, Einstein obtém as leis de conservação de sua teoria, mostrando a conservação covariante do tensor energia-momento. Na seção seguinte, Einstein analisa alguns casos particulares.

\section{Fenômenos de matéria}

Na quarta seção, Einstein mostra que a hidrodinâmica e o eletromagnetismo, como estudados na relatividade restrita, podem ser descritos com o aparato da relatividade geral. Em particular, o caso da hidrodinâmica é estendido para o contexto do espaço curvo, enquanto o eletromagnetismo é considerado apenas na ausência de campos de gravitação.

\section{E. Limite newtoniano e consequências observacionais}

A última seção do artigo não é nomeada e o título acima é emprestado de Tilman Sauer [33], pois reflete bem o conteúdo da seção. Einstein analisa o limite de campo fraco de sua teoria, mostrando que a teoria newtoniana surge como o limite da relatividade geral: a componente $g_{44}$ da métrica (na notação de Einstein, e que aqui posteriormente será chamada de $g_{00}$ ), no referido limite, faz o papel do potencial gravitacional newtoniano,

$$
\nabla^{2} g_{44}=\kappa \rho
$$

No caso de uma massa puntiforme, como mostrado pelo próprio Einstein, o potencial gravitacional pode ser entendido como a primeira ordem de perturbação à métrica de Minkowski em que

$$
g_{44}=1-\frac{\alpha}{r}
$$

A partir desse resultado, Einstein discute como as medidas de comprimento e tempo são alteradas na presença de gravitação e como o desvio de periélio e a deflexão dos raios de luz são obtidos no contexto da nova teoria.

Como curiosidade, é interessante observar que, para a previsão para a deflexão da luz, apenas a correção de primeira ordem no tensor métrico é suficiente para prevê-la com boa precisão. Ou seja, Einstein ainda não possuía uma solução analítica sem aproximação, mas sua teoria já era suficiente para fazer novas previsões. No entanto, para a descrição do avanço de periélio é necessário considerar termos não lineares - conforme mencionado por 
Einstein no artigo de 1916, a solução foi estudada por ele próprio em 1915 e confirmada no contexto da solução de Schwarzschild em 1916. Esta, por sua vez, foi a primeira solução exata para as equações de Einstein e um ponto interessante é que, a partir da solução exata, também é possível fazer previsões a respeito do desvio da luz com a mesma precisão que a solução aproximada. Essa análise será feita na próxima seção.

\section{Deflexão da luz numa versão moderna da relatividade geral}

Os primeiros testes da relatividade geral, o que inclui a deflexão da luz, podem ser obtidos da primeira solução analítica das equações de campo. Trata-se de uma abordagem moderna, repleta de consequências astrofísicas e cosmológicas.

Em 1916, Karl Schwarzschild introduziu a métrica, ou seja, o elemento de linha infinitesimal no espaço curvo quadridimensional, dada por [34]

$$
\begin{aligned}
d s^{2} & =\left(1-\frac{2 G M}{c^{2} r}\right) c^{2} d t^{2}-\left(1-\frac{2 G M}{c^{2} r}\right)^{-1} d r^{2} \\
& -r^{2}\left(d \theta^{2}+\operatorname{sen}^{2} \theta d \varphi^{2}\right)
\end{aligned}
$$

que representa um objeto de massa $M$, esfericamente simétrico e estático. Essa solução prevê, pela primeira vez, a existência de objetos exóticos que vieram a ser chamados de buracos negros. De fato, em rápida análise, nota-se que existem duas regiões de singularidade nessa solução: uma singularidade física em $r=0$ e outra singularidade puramente matemática em $r_{g}=2 G M / c^{2}$, conhecida como raio gravitacional, ou raio de Schwarzschild. Esse resultado faz menção à existência de uma superfície esférica conhecida como horizonte de eventos do buraco negro, de onde nada escapa, nem mesmo a luz. No entanto, o objetivo aqui é abordar o problema da deflexão da luz.

\subsection{Equação de trajetória}

Para a obtenção das equações de trajetória de um feixe de luz próximo a uma fonte de matéria, tal qual o Sol, fazse necessário o cálculo das equações geodésicas (ver Eq. (51)) para o caso da solução de Schwarzchild. Para tanto, considerando o elemento de linha da Eq. (58), sua relação direta com os componentes do tensor métrico, $g_{\mu \nu}$, como mostrado na Eq. (52), e considerando as componentes $x^{0}=t x^{1}=r x^{2}=\theta x^{3}=\varphi$ é suficiente calcular cada um dos componentes do símbolo de Christoffel, como mostrado na Eq. (53), e substituí-los na equação geodésica. Trata-se de uma tarefa que exige paciência e certo trato com a notação utilizada na relatividade geral. Contudo, o desenvolvimento das operações é descrito na maioria dos livros introdutórios de relatividade geral [35]. Aqui as equações da geodésicas serão meramente transcritas na forma 17

$$
\begin{gathered}
\theta: \frac{d^{2} \theta}{d s^{2}}+\frac{2}{r} \frac{d r}{d s} \frac{d \theta}{d s}-\cos \theta \operatorname{sen} \theta\left(\frac{d \varphi}{d s}\right)^{2}=0 \\
\varphi: \frac{d^{2} \varphi}{d s^{2}}+\frac{2}{r} \frac{d r}{d s} \frac{d \varphi}{d s}=0 \\
t: \frac{d^{2} t}{d s^{2}}+\frac{G M}{c^{2} r^{2}}\left(1-\frac{2 G M}{c^{2} r}\right)^{-1} \frac{d r}{d s} \frac{d t}{d s}=0 .
\end{gathered}
$$

Usando o resultado da relatividade restrita que o elemento de linha de um raio de luz é $d s^{2}=0$, a Eq. (58) torna-se

$$
\begin{aligned}
& \left(1-\frac{2 G M}{c^{2} r}\right) c^{2} d t^{2}-\left(1-\frac{2 G M}{c^{2} r}\right)^{-1} d r^{2} \\
& -r^{2}\left(d \theta^{2}+\operatorname{sen}^{2} \theta d \varphi^{2}\right)=0 .
\end{aligned}
$$

Uma análise da equação geodésica para a componente em $\theta$ permite concluir que o movimento deve ocorrer sob um plano fixo. Para tanto, basta assumir, sem perda de generalidade, as seguintes condições iniciais: $\theta_{t=0}=\frac{\pi}{2}$, $\left(\frac{d \theta}{d s}\right)_{t=0}=0$, que resultam em $\frac{d^{2} \theta}{d s^{2}}=0$, em qualquer instante do tempo. Ou seja, entre as condições consideradas, o movimento fica confinado no plano $\theta=\frac{\pi}{2}$, como pode ser visto na Fig. 10.

$\mathrm{E}$ as equações geodésicas para $\varphi$ e $t$ podem ser reduzidas a

$$
r^{2} \frac{d \varphi}{d s}=b \quad \text { e } \quad \frac{d t}{d s}=k \cdot\left(1-\frac{2 G M}{c^{2} r}\right)^{-1}
$$

em que $k$ e $b$ são constantes de integração. Importante mencionar que a integração da equação geodésica para $\varphi$, resultando em uma constante, está diretamente relacionada à conservação do momento angular, como esperado, já que a configuração do sistema é exatamente a mesma da de um corpo sob a ação de uma força central. Substituindo, de forma adequada, a Eq. (61) na Eq. (60),

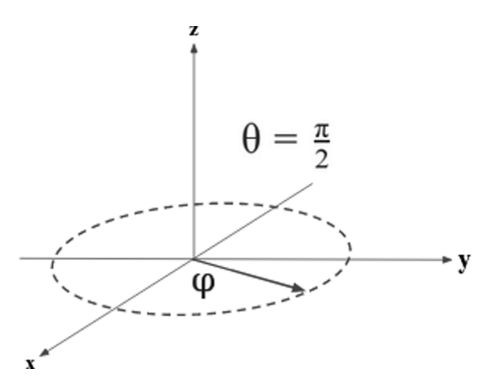

Figura 10: A linha tracejada representa a trajetória de uma partícula na presença de uma quantidade de matéria centrada no eixo coordenado. Notem que a partícula fica confinada no plano $(x y)$, que corresponde ao ângulo zenital $\theta=\frac{\pi}{2}$.

\footnotetext{
17 A equação para a componente $r$ é omitida porque não costuma ser usada no desenvolvimento dos cálculos, havendo itinerários que simplificam o trabalho na direção do objetivo final.
} 
mudando para a variável $u=\frac{1}{r}$ e derivando toda a equação com relação a $\varphi$, chega-se a

$$
\frac{d^{2} u}{d \varphi^{2}}+u=\frac{3 G M}{c^{2}} u^{2}
$$

que é a equação de trajetória de uma partícula nas proximidades de uma quantidade de matéria simetricamente esférica e estática, que curva o espaço-tempo, na métrica de Schwarzschild.

\subsection{Solução da equação de trajetória}

Na solução da Eq. (62), utiliza-se o método de aproximações sucessivas, como nas teorias de perturbação. Esse método consiste em tomar uma solução geral como uma série de potências

$$
u=u^{(0)}+u^{(1)}+u^{(2)}+\ldots
$$

em que, para solução de $u^{(1)}$ utiliza-se $u^{(0)}$, de $u^{(2)}$ utilizase $u^{(1)}$, assim sucessivamente, até a ordem pretendida. Neste trabalho são apresentadas soluções em primeira ordem, já que outras ordens pouco interferem na precisão do resultado em comparação com os valores experimentais. Assim, em primeira ordem, a solução é dada por

$$
u=\frac{1}{R} \cos \varphi+\frac{G M}{R^{2}}\left(\cos ^{2} \varphi+2 \operatorname{sen}^{2} \varphi\right) .
$$

ou

$$
R=r \cos \varphi+\frac{G M}{c^{2} R}\left(r \cos ^{2} \varphi+2 r \operatorname{sen}^{2} \varphi\right)
$$

que, em coordenadas cartesianas, pode ser escrita como

$$
x=R-\frac{G M}{c^{2} R} \frac{x^{2}+2 y^{2}}{\sqrt{x^{2}+y^{2}}} .
$$

Suponha que o feixe de luz venha de uma fonte muito distante, tal que $y \gg x$, como mostrado na Fig. 11. Nesse caso, é boa a aproximação

$$
x \approx R \mp \frac{G M}{c^{2} R} \cdot 2 y
$$

que representa a equação de duas retas de coeficiente angular $m_{+}=\frac{2 G M}{c^{2} R}$ e $m_{-}=-\frac{2 G M}{c^{2} R}$. Portanto, a trajetória da luz é dada pela composição dessas duas soluções, em que as respectivas retas tangenciam o percurso da luz, como mostrado na Fig. 11. O desvio na trajetória da luz é determinado pelo ângulo $\delta=2 \omega$. O resultado final, previsto por Einstein, é

$$
\delta=\frac{4 G M}{c^{2} R} \approx 1,75^{\prime},
$$

que vale o dobro do valor newtoniano calculado por Soldner e Cavendish.

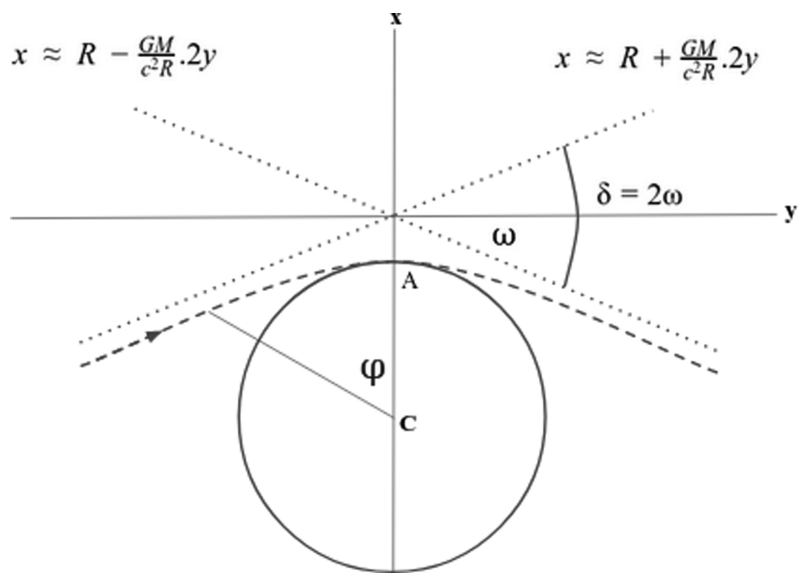

Figura 11: A linha tracejada representa a trajetória da luz, enquanto as duas retas de linha pontilhada representam a solução da equação da trajetória. Note que o trajeto do feixe de luz tangencia as duas retas. Portanto, o desvio devido à ação do campo gravitacional se dá pelo ângulo entre estas, que, no caso, é igual a $\delta=2 \omega$.

\section{Referências}

[1] I.B. Cohen, Isaac Newton's Papers $\& 3$ Letters on Natural Philosophy and related documents (Harvard University Press, Cambridge, 1958).

[2] I. Newton, Óptica, Trad. André Assis (Editora da Universidade de São Paulo, São Paulo, 2002).

[3] A. Raftopoulos, N. Kalyfommatou e C.P. Constantinou, Science \& Education 14, 649 (2005).

[4] C.C. Silva e B.A. Moura, Revista Brasileira de Ensino de Física 30, 1602 (2008).

[5] J.P. Marat, Découvertes sur la lumière (Jombert, Paris, 1780).

[6] D. Valls-Gabaud, Cosmology Across Cultures ASP Conference Series 409, 57 (2009).

[7] J.P. Marat, Notions élémentaires d'optique (Hachette Livre BNF, Paris, 1784).

[8] W.A. Osborne, Nature 105, 456 (1920).

[9] C.D. Conner, Jean-Paul Marat, scientist and revolutionary (Humanity Books, New Jersey, 1997).

[10] J.J. Thomson, The Observatory 42, 389 (1919).

[11] S.I. Newton, Opticks; or A treatise of the reflections, refractions, inflections and colours of light (Dover, New York, 1952).

[12] R. McCormmach, The British Journal for the History of Science 4, 126 (1968).

[13] J. Michell, Philosophical Transactions 74, 35 (1784).

[14] J. Eisenstaedt, Archive for History of Exact Sciences 42, 315 (1991).

[15] R. McCormmach, Weighing the world: the reverend John Michell of Thornhill (Springer, Dordrecht, 2012).

[16] J.G. von Soldner, Astronomisehes Jahrbuch für das Jahr 1804 (C.F.E. Spfithcn, Berlin, 1801), p. 161.

[17] S.L. Jaki. Foundations of Physics 8, 927 (1978).

[18] P. Lenard. Annalen der Physik 65, 593 (1921).

[19] H. Cavendish, The scientific papers of the honourable Henry Cavendish, F.R.S., Vol. II: Chemical and Dynamical (E. Thorpe, Cambridge, 1921). 
[20] F. Dyson (1921), Unpublished papers on astronomical subjects. On Cavendish's astronomical papers. Ref. [19, p. 433].

[21] C.M. Will, American Journal of Physics 56, 413 (1988).

[22] C. Jungnickel e R. McCormmach, Cavendish, disponível em http://bit.ly/2IAUdyq.

[23] H. Goldstein, C.P. Poole e J.L. Safko, Classical mechanics (Addison Wesley, Massachusetts, 2001).

[24] A. Einstein, Physics Today 35, 45 (1982).

[25] A. Einstein, Revista Brasileira de Ensino de Física 27, 37 (2005).

[26] https://einsteinpapers.press.princeton.edu/vol7-trans/129.

[27] A. Einstein, Annalen der Physik 35, 898 (1911).

[28] A. Einstein, em: $O$ ano miraculoso de Einstein, organizado por J. Stachel (Editora UFRJ, Rio de Janeiro, 2001).

[29] S. Paolantonio, Rev. Bras. Ens. Fis. 41, e20190206 (2019).

[30] A. Einstein, Annalen der Physik 49, 769 (1916).

[31] A. Pais, Subtle is the Lord... The science and the life of Albert Einstein (Clarendon Press, Oxford, 1982).

[32] A. Einstein, The origins of the general theory of relativity (Jackson, Wylie and Co., Glashow, 1933).

[33] T. Sauer, arXiv:0405066v1 (2004).

[34] K. Schwarzschild, arXiv:9905030 (1999).

[35] V. Sabbata e M. Gasperini Introduction to gravitation (World Scientific Publishing Co., Singapore, 1985). 